\title{
Symmetry Approach to Extension of Flutter Boundaries via Mistuning
}

\author{
B. Shapiro* \\ California Institute of Technology, Pasadena, California 91125
}

\begin{abstract}
A general framework is presented for analyzing and optimizing stability increases resulting from mistuning. The framework given is model independent and is based primarily on symmetry arguments. Difficult practical issues are transformed to tractable mathematical questions. It is shown that mistuning analysis reduces to a block circular matrix eigenvalue/vector problem that can be solved efficiently even for large problems. Similarly, the optimization becomes a standard linear constraint quadratic programming problem and can be solved numerically. Because the methods given are model-independent, they can be applied to various models and allow the researcher to easily conclude which models accurately capture mistuning and which do not. A simple quasisteady model for flutter in a cascade is used to illustrate and validate results in this paper.
\end{abstract}

\section{Nomenclature}

$a \quad=$ linear stability coefficient, Eqs. (13), (14), and (35)

$b \quad=$ diagonal quadratic stability coefficient, Eqs. (13), (14), and (36)

$c_{i} \quad=i$ th off-diagonal quadratic stability coefficient, Eqs. (13), (14), and (37)

$\boldsymbol{f} \quad \boldsymbol{=} \boldsymbol{f}(\boldsymbol{x}, U, \boldsymbol{z})$, flutter model, Eq. (1)

$h \quad=h(U, z)$, negative of minimum damping, Eq. (3)

$k=r / 2$ when $r$ even, $(r-1) / 2$ if $r$ odd

$M \quad=M(U, z)$, linearization matrix in Eq. (15)

$m \quad=$ number of states per blade

$r \quad=$ number of blades

$S \quad=$ stability extension matrix, Eq. (14)

$s \quad=s(z)$ stability extension, Eq. (5)

$U \quad=$ loading parameter in Eq. (1)

$U_{\text {crit }}=U_{\text {crit }}(z)$, instability inception loading, Eq. (4)

$\boldsymbol{U}_{i}, \boldsymbol{V}_{i}=i$ th left and right eigenvectors of $M\left[U_{\text {crit }}(0), 0\right]$ in Eqs. (38) and (39)

$\boldsymbol{x}=\left(\boldsymbol{x}_{1}, \boldsymbol{x}_{2}, \ldots, \boldsymbol{x}_{r}\right)$ state vector, $\boldsymbol{x}_{i} \in \mathbb{R}^{m}$ state of $i$ th blade, Eq. (1)

$x_{0}=x_{0}(U, z)$, relevant equilibrium point of model, Eq. (1)

$\boldsymbol{z}=\left(z_{1}, z_{2}, \ldots, z_{r}\right)$ mistuning vector, $z_{i} \in \mathbb{R}$ mistuning of $i$ th blade, Sec. II

$\bar{z}, z^{*}=$ optimal linear and quadratic solutions

$\boldsymbol{\alpha}^{*} \quad=$ beneficial mistuning crossover, Eq. (48) and Fig. 6

$\lambda_{j}=\lambda_{j}(U, z)$ is $j$ th eigenvalue of linearization $M(U, z)$, Eq. (15)

$\lambda_{p} \quad=$ least-stable eigenvalue

$\boldsymbol{\varsigma}=$ rotation operator, Assumption 2

$=$ transpose of vector or matrix

\section{Introduction}

$\mathbf{J}$ ET-engine performance is severely limited by a wide variety of instabilities, including inlet buzz, shear-layer turbulence, and compression instabilities (Fig. 1). The ability to eliminate or reduce the severity of these instabilities can lead to increased safety, higher efficiency, and significant cost and weight savings in future engine designs. This work focuses on

Received April 28, 1997; revision received Nov. 16, 1997; accepted for publication Nov. 24, 1997. Copyright (C) 1998 by the American Institute of Aeronautics and Astronautics, Inc. All rights reserved.

*Graduate Student, Control and Dynamical Systems. E-mail: bshapiro@indra.caltech.edu. flutter in compression systems because it is encountered in every regime operation and clearly limits engine performance (Fig. 2). ${ }^{1}$ The interested reader is referred to Ref. 2 for a general overview of turbomachinery instabilities.

In this paper we study passive control of flutter using mistuning. In this context, mistuning refers to symmetry breaking. For example, all of the blades in a spinning fan or bladed disc are nominally identical, and so there exists a discrete circumferential symmetry. It has been noted numerically ${ }^{3-5}$ and experimentally ${ }^{6}$ that if we mistune these blades by making them different from one another, then the flutter boundary can be delayed dramatically. Techniques presented herein restrict attention to circumferential symmetry breaking but apply to any type of instability. We use flutter in compression systems as a concrete example and note that the same methods hold for other instabilities such as rotating stall or surge.

Experiments have shown that mistuning rotor stiffness in compression systems not only increases the range of stability but also creates undesirable side effects such as mode localization $^{7-9}$ and decreased operating range. ${ }^{10}$ This leads to two natural questions:

1) Analysis: For a given mistuning find new stability boundaries and resulting side effects.

2) Synthesis: When is mistuning beneficial? If it is, find optimal mistuning (increase stability with acceptable side effects).

Variations on the analysis issue have been addressed in past research. Dugundi and Bundas ${ }^{11}$ use Whitehead's aerodynamic coefficients ${ }^{12}$ to predict the stability increase for alternate blade mistuning. Bloemhof ${ }^{13}$ considers stability increases caused by single-, double-, and triple-blade alternate mistuning along with aperiodic or random mistuning. Regarding the second part of the analysis question, we note that most work on predicting side effects as a function of mistuning has focused on mode localization. Papers in this area include Refs. 5, 9, 11, 14, and 15 . Work on related side effects such as decreased operating range can be found in Srinivasan and Frye. ${ }^{10}$ However, efficient solutions to the synthesis issue do not exist at this time mainly because there are no efficient solutions of the analysis problem for arbitrary mistuning. Crawley and $\mathrm{Hall}^{4}$ and Nissim and $\mathrm{Haftka}^{16}$ tackle an optimization problem where they minimize the size of mistuning (implicitly assuming that resulting side effects are also minimized) subject to a required stability increase. Even though this is a reasonable approach, we note 


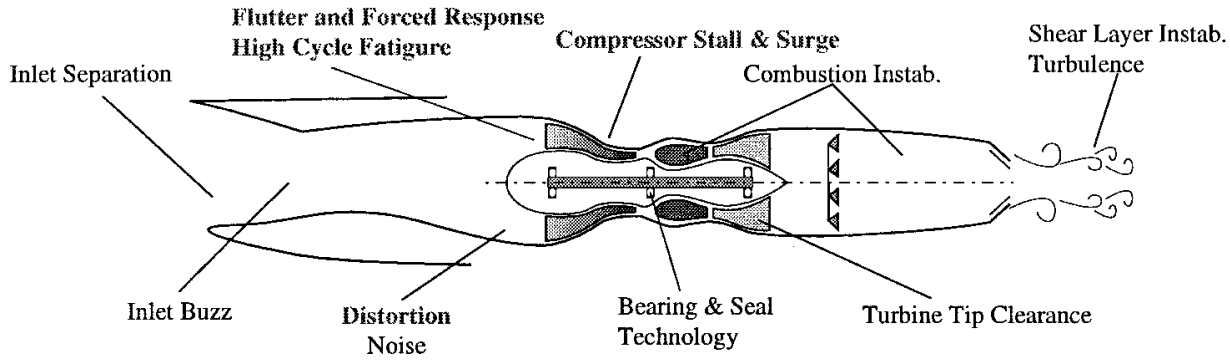

Fig. 1 Turbomachinery instabilities (figure courtesy of J. Paduano).

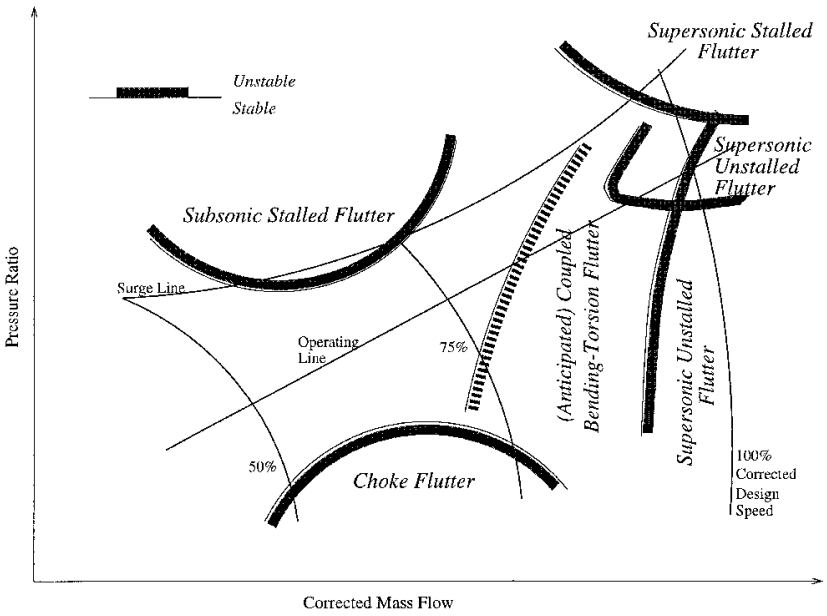

Fig. 2 Compressor map slowing typical flutter boundaries. ${ }^{1}$

that in both papers only local optimums are found and both methods are restricted to small problems (less than 14 blades) by lengthy computation times.

Furthermore, previous mistuning work has not made use of symmetry arguments to aid in the analysis. Yet mistuning is concerned primarily with symmetry and symmetry breaking, and so symmetry arguments form the natural tools for the mistuning problem. Using simple symmetry arguments that apply to any model type, whether it is computational fluid dynamics (CFD), sinusoidal imposed motion, or dynamical system, simplifies the analysis problem tremendously. Finding the stability boundary as a function of arbitrary mistuning reduces to finding $r / 2+2$ stability coefficients. One then requires a specific model to evaluate the $r / 2+2$ stability coefficients.

We give two methods to compute these stability coefficients. Method A (Sec. IV.A) is valid for any dynamical systems model of the form $\dot{\boldsymbol{x}}=\boldsymbol{f}(\boldsymbol{x})$, and method B (Sec. IV.B) is practical for CFD models of small to moderate size. Method A relates the stability coefficients to a block circular matrix eigenvalue/vector problem that can be solved efficiently even for large problems, whereas method B involves the finite difference of CFD stability results. Future research could lead to further methods that would compute the stability coefficients for large CFD codes and imposed sinusoidal motion models.

Our focus is on dynamical system models as opposed to imposed sinusoidal motion models because it is more natural to study mistuning in the former case. In particular, imposed motion models usually assume constant interblade phase angle. Although this assumption is valid in the tuned case, upon mistuning the eigenvectors (which correspond to the imposed motion), are perturbed away from the symmetric case, and so the constant interblade phase angle assumption is violated. Our analysis of unconstrained dynamical system models avoids these difficulties.

We note that the same model-independent symmetry arguments used in the analysis can be used to reduce the optimization problem to a standard quadratic programming problem with constraints. In this paper we simply restrict the maximal size of allowable mistuning and assume that the resulting side effects are acceptable. Consequently, the optimization becomes a linear constaint quadratic program. Using branch and bound software developed by Faiz et al. ${ }^{17}$ it is possible to find global optimums for fairly large problems (up to about 30 blades). More realistic constraints motivated by side effects in general and mode localization in particular shall be addressed in future research. Finally, to further illustrate the power of symmetry arguments we show that with additional structure found in the quasisteady model of Appendix A, the (different) optimal arrangement problem of Sec. VI may be solved closed-form.

\section{Problem Setup}

As motivation, consider a compressor fan with $r$ blades. Nominally, all of the blades are identical and there exists a $2 \pi / r$ circumferential symmetry. Similarly, stators, struts, and inlet guide vanes (IGVs) can also possess circumferential symmetries. Given such a tuned system with $r$ discrete objects, such as blades, stators, or IGVs, and a $2 \pi / r$ circumferential symmetry begin by defining a mistuning vector $z \in \mathbb{R}^{r}$. An element $z_{i}$ denotes mistuning for the $i$ th object. For example, if we mistune the stiffness of $r$ rotors, then define the $i$ th blade stiffness $k_{i}=k_{0}\left(1+z_{i}\right)$, where $k_{0}$ is the nominal or tuned stiffness. In general, we will define $z$ so that $z=0$ corresponds to the tuned case.

Our next goal is to define the increase in stability boundary $s(z)$ caused by mistuning. To do this in a precise way consider a dynamical system model of the following form:

$$
\dot{x}=f(x, U, z)
$$

where $\boldsymbol{x} \in \mathbb{R}^{n}$ is the state vector, $U \in \mathbb{R}$ is a loading parameter such as throttle, Mach number, reduced frequency, or rotor speed, and $z$ is the previously defined mistuning vector. In this context, $f$ can be any discrete blade model or even a CFD model. It is stressed that although we focus on dynamical system models [Eq. (1)], the symmetry arguments presented apply more generally. These arguments are valid for any model with symmetry, symmetry breaking, and a stability boundary $s(z)$. In particular, the symmetry methods apply to imposed sinusoidal motion models.

For a fixed $z$, as we vary the loading parameter $U$ within some operating range $\left[U_{0}, U_{1}\right]$, the system [Eq. (1)] traverses a set of equilibria $X_{0}(U, z)$ defined by

$$
X_{\mathrm{o}}(U, \boldsymbol{z})=\left\{\boldsymbol{x}_{\mathrm{0}}: \boldsymbol{f}\left(\boldsymbol{x}_{\mathrm{0}}, U, \boldsymbol{z}\right)=0, U \in\left[U_{0}, U_{1}\right]\right\}
$$

Assuming that $X_{0}(U, z)$ is nonempty for $z$ in some neighborhood of the origin and for all $U \in\left[U_{0}, U_{1}\right]$, we choose a subset $x_{0}(U, z) \subset X_{0}(U, z)$ that corresponds to the equilibrium point of interest at each $U$. As an example, suppose we have a model [Eq. (1)] of a (tuned) jet engine with $z=0, U$ is the throttle and it varies between $U_{0}$ and $U_{1}$, then we can think of $\boldsymbol{x}_{0}(U$, $0)$ as the design operating point that varies as a function of throttle setting. Clearly, $\boldsymbol{x}_{0}: \mathbb{R} \times \mathbb{R}^{r} \rightarrow \mathbb{R}^{n}$ is a function of $U$ and $z$, possibly discontinuous and nonsmooth in both arguments. Continuity and smoothness assumptions for $\boldsymbol{x}_{0}(U, \boldsymbol{z})$ will be discussed in the next section. It is important to realize 


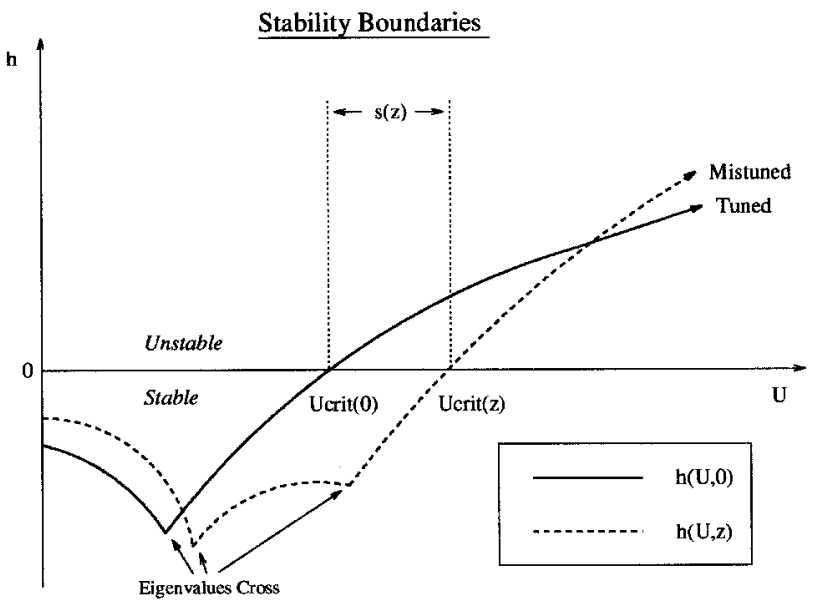

Fig. 3 Increase in stability boundary, $s(z)$.

that the $z$ dependence in $\boldsymbol{x}_{0}(U, z)$ is essential. Consider once again stiffness mistuning: because different blades have different stiffnesses, their nominal (or static) deflections are no longer equal, which implies that the mistuned equilibrium point is not equal to the tuned equilibrium point $\left[x_{0}(U, z) \neq\right.$ $x_{0}(U, 0)$ for $\left.z \neq 0\right]$.

Now consider the stability of $\boldsymbol{x}_{0}(U, z)$ as a function of $U$ for a fixed $z$. Define

$$
h(U, z)=\max \{\operatorname{Re}[\boldsymbol{\lambda}(U, \boldsymbol{z})]\}
$$

where $\lambda(U, z)$ are the eigenvalues of $(\partial f / \partial x)\left[x_{0}(U, z), U, z\right]$. If $h(U, z)$ is negative (respectively positive), then the equilibrium point $\boldsymbol{x}_{\mathrm{o}}(U, \boldsymbol{z})$ is stable (respectively unstable). Because we are concerned with stability, it is assumed that as the loading $U$ increases, then at some point $U_{\text {crit }}$ stability is lost. Thus, define

$$
U_{\text {crit }}(\boldsymbol{z})=\min _{u \in\left[U_{0}, U_{1}\right]}\{u: h(u, z)=0\}
$$

If $h(U, z)$ does not cross the origin for $U \in\left[U_{0}, U_{1}\right]$ then let $U_{\text {crit }}(z)= \pm \infty$ with the appropriate choice of sign. (When the system loses stability as $U$ decreases the min should be replaced with a max. Also, if there is more then one stability boundary of interest, the interval $\left[U_{0}, U_{1}\right]$ may be appropriately partitioned so that only one boundary is under consideration.) Finally, the increase in stability $s(z)$ is defined as

$$
s(z)=+\left[U_{\text {crit }}(z)-U_{\text {crit }}(0)\right]
$$

where the positive sign is replaced with a negative sign if instability occurs as $U$ decreases. Physically, loading $U$ may be time dependent, if we take $U$ as a Mach number then it varies with outside disturbances and as the blades sweep past stators and inlet guide vanes. However, we can always split a time-dependent loading $U(t)$ into an averaged and perturbed part: $U(t)=U+U^{\prime}(t)$. The steady part $U$ leads to the study of stability (our current focus) while the unsteady part $U^{\prime}(t)$ is exactly the forced response or mode localization problem (the subject of future work).

To summarize, $s(z)$ is simply the change in stability (at the relevant equilibrium point) as a function of mistuning. The preceding technical remarks are appropriate because they allow us to equate assumptions on the smoothness of $s$ with smoothness conditions on $f$. For a graphical interpretation see Fig. 3.

\section{Assumptions}

Two basic assumptions are required for the analysis that follows.
Assumption 1 (smoothness): The stability extension $s(z)$ is three times differentiable in some sufficiently large neighborhood of the origin, $s \in \mathrm{C}^{3}(\Omega)$.

Smoothness is necessary so that we may take derivatives with respect to $z$. We also require that the analysis holds in some sufficiently large region $\Omega$ about the origin (tuned case) so that predicted shifts in stability hold for a physically practical range of mistuning values $z$. Note that assumption 1 is a technical condition that can be relaxed in a more detailed, but equally straightforward, analysis. Such an extension typically requires the tracking of multiple eigenvalues.

Assumption 1 is easy to check if Eq. (1) is simple. As a concrete example, Assumption 1 can be verified for the quasisteady model used later in this paper. More generally, some fairly weak assumptions (which may be difficult to check), on the original model $\dot{\boldsymbol{x}}=\boldsymbol{f}(\boldsymbol{x}, U, \boldsymbol{z})$ imply assumption 1 . We briefly mention three cases that can violate assumption 1 .

Case 1 occurs if the equilibrium point $x_{0}(U, z)$ does not travel smoothly with $U$ and/or $z$. Typically, such problems are caused by equilibrium bifurcations and present a host of difficulties that must be dealt with before stability can be considered. Case 2 comes about if $h(U, 0)$ has a degenerate root at $U_{\text {crit }}(0)$ as shown in Fig. 4. Here $s(z)$ is discontinuous in $z$ and assumption 1 is violated. Under such circumstances one would consider the minimum damping instead of the stability extension $s(z)$. Case 3 illustrates a possible restriction on the second part of assumption 1. If the real part of the least stable (or critical eigenvalue) in $h(U, 0)$ is very close to the real part of another eigenvalue, then it is possible that these real parts will switch as $z$ is varied (Fig. 5).

In this case $s(z)$ will be smooth on a very small region and the second part of assumption 1 is broken. For a nondistinct least-stable eigenvalue we have the special case where $s(z)$ can be discontinuous at the origin. It is possible to avoid these problems by keeping track of a number of eigenvalues during the analysis.

Assumption 2 captures the symmetry of the problem and is the main driving force behind the analysis.

Assumption 2 (symmetry): The minimum damping $h(U, z)$, and hence the stability extension $s(z)$, are invariant under rotations of $z$. Specifically

$$
\begin{gathered}
h(U, z)=h\left(U, \varsigma^{k}[z]\right), \quad \forall U, z, \quad \forall k \in(1,2, \ldots, r-1) \\
\Rightarrow s(z)=s\left(\varsigma^{k}[z]\right), \quad \forall z, \quad \forall k \in(1,2, \ldots, r-1)
\end{gathered}
$$

where $\varsigma^{k}\left[z_{1}, z_{2}, \ldots, z_{r}\right]=\left[z_{1+k}, z_{2+k}, \ldots, z_{r}, z_{1}, \ldots, z_{k}\right]$ is the rotation operator.

This is the symmetry group discussed in Secs. I and II. In physical terms: The system should exhibit identical behavior if we mistune the $i$ th (say first) or jth (say third) blade (object). Specifically, the stability boundary must remain the same,

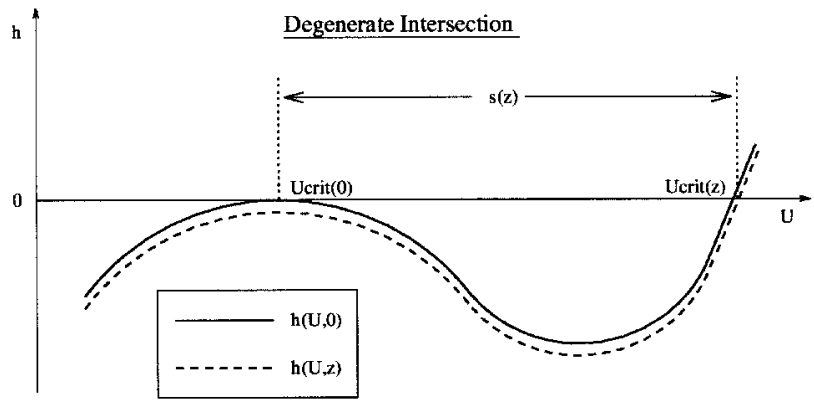

Fig. 4 Discontinuous increase in stability boundary. 


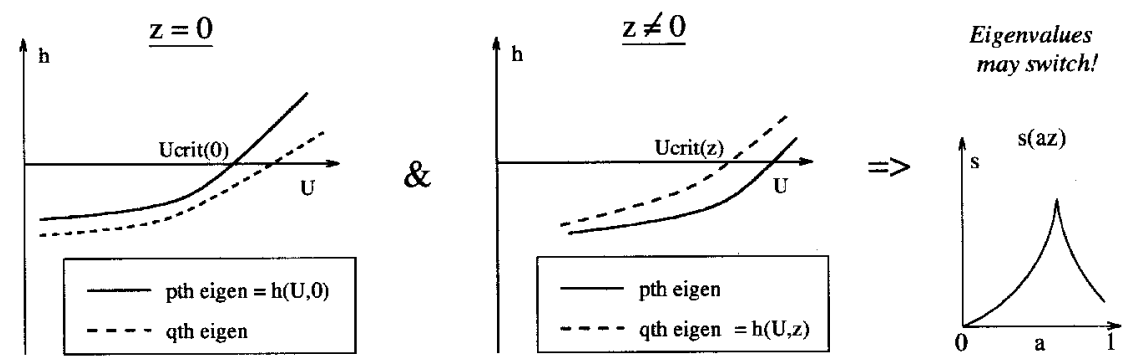

Fig. 5 Eigenvalue switching creates a discontinuity in $s(z)$.

hence $s(\varepsilon, 0, \ldots, 0)=s(0,0, \varepsilon, 0, \ldots, 0)$. Alternatively, a rotation of $z$ corresponds to a circular renumbering of blades. Because labeling schemes are arbitrary, it follows that stability cannot be changed by such renumbering, and so, $s(z)$ is invariant under rotation. In spite of its simplicity, assumption 2 will yield a surprising amount of analytical mileage.

Of course, perfect tuned bladed disk assemblies, do not exist in practice, and so, strictly speaking, assumption 2 fails. However, we may consider a practical fan as a small perturbation $\boldsymbol{\varepsilon}$ of the nominal or theoretically perfect fan. Upon applying mistuning to this realistic fan, we introduce a larger intentional perturbations $\boldsymbol{\xi}$ for a total mistuning $\boldsymbol{z}=\boldsymbol{\varepsilon}+\boldsymbol{\xi}$. Assumption 2 now applies to $z$. It will be shown in the next section that mistuning appears as a second-order effect (possibly with large quadratic coefficients), and so, sufficiently small (zero average) imperfections $\varepsilon$ produce negligible stability effects. The size of sufficiently small depends on the ratio $\boldsymbol{\alpha}^{*}$ of linear to quadratic coefficients [see Section V, Eq. (48)]. Finally, note that assumption 2 holds for most practical models, in particular it holds for models in all of the mistuning articles cited in this paper.

\section{Analysis}

In this section we address the analysis problem: given a mistuning $z$ find the related stability extension $s(\boldsymbol{z})$. Mode localization caused by mistuning will be addressed in future work. At present, we restrict the size of mistuning (as described in the next section) and assume that the resulting mode localization is acceptable.

Our goal is to determine the form of $s(z)$. By assumption 1, we can expand $s(z)$ in a power series about the origin

$$
s\left(z_{1}, \ldots, z_{r}\right)=\sum_{i=1}^{r} a_{i} z_{i}+\sum_{i, j=1}^{r} b_{i j} z_{i} z_{j}+\mathcal{O}\left(\|z\|^{3}\right)
$$

The power expansion holds in a region no bigger than $\Omega$ - the range where $s(z)$ is smooth. Let $\Theta \subset \Omega$ be the subset where $s(z)$ is accurately approximated by second-order terms in $z$. It is additionally assumed that this smaller set $\Theta$ is still sufficiently large to be of practical interest. If this is not the case, the analysis can be extended to include third- and fourth-order terms at the expense of increased complexity and computation time.

By assumption 2, $s(z)=s\left(\varsigma^{k}[z]\right)$ for all integers $k$. Pick $z=$ $[\varepsilon, 0, \ldots, 0]$ and substitute $s^{k}[z]$ into the power expansion for all $k \in(0,1,2, \ldots, r-1)$ to obtain

$$
a_{1} \varepsilon+b_{11} \varepsilon^{2}=a_{2} \varepsilon+b_{22} \varepsilon^{2}=\cdots=a_{r} \varepsilon+b_{r r} \varepsilon^{2}
$$

which holds for all $\varepsilon$ up to $\mathcal{O}\left(\varepsilon^{3}\right)$. Consequently

$$
\begin{aligned}
& a_{i}=a_{j} \\
& b_{i i}=b_{j j}
\end{aligned}
$$

for all $i$ and $j$. Similarly, let $z=[0, \ldots, 0, \varepsilon, 0, \ldots, 0, \varepsilon, 0$, $\ldots, 0]$, where the $\varepsilon s$ are located in the $i$ th and $j$ th spots, by varying $i, j$, and $k$, we can show

$$
b_{i j}+b_{j i}=b_{[i+k][j+k]}+b_{[j+k][i+k]}
$$

for all $i, j$, and $k$, where $[i+k]=(i+k) \bmod r$. Hence, we make the following definitions:

$a \triangleq a_{1}=a_{2}=\cdots=a_{r}$

$b \triangleq b_{11}=b_{22}=\cdots=b_{r r}$

$c_{1} \triangleq b_{12}+b_{21}=b_{23}+b_{32}=\cdots=b_{[r-1] r}+b_{r[r-1]}=b_{r 1}+b_{1 r}$

$c_{2} \triangleq b_{13}+b_{31}=b_{24}+b_{42}=\cdots=b_{[r-1] 1}+b_{1[r-1]}=b_{r 2}+b_{2 r}$

$$
\text { : } \quad \text { : }
$$

$c_{k} \triangleq b_{1[1+k]}+b_{[1+k] 1}=\cdots=b_{r k}+b_{1 k}$

where $k$ is defined in the remainder as

$$
k \triangleq\left\{\begin{array}{lll}
r / 2 & r & \text { even } \\
(r-1) / 2 & r & \text { odd }
\end{array}\right.
$$

Using the preceding definitions, we can rewrite Eq. (8) as

$$
\begin{aligned}
s(\boldsymbol{z})= & a\left(z_{1}+z_{2}+\cdots+z_{r}\right) \\
& +b\left(z_{1}^{2}+z_{2}^{2}+\cdots+z_{r}^{2}\right) \\
& +c_{1}\left(z_{1} z_{2}+z_{2} z_{3}+\cdots+z_{r} z_{1}\right) \\
& +c_{2}\left(z_{1} z_{3}+z_{2} z_{4}+\cdots+z_{r} z_{2}\right) \\
& \vdots \\
& +c_{k}\left(z_{1} z_{1+k}+z_{2} z_{2+k}+\cdots+z_{r} z_{k}\right)+\mathcal{O}\left(\|z\|^{3}\right)
\end{aligned}
$$

It is obvious by inspection that $s(z)$ is invariant under rotation as advertised. Observe that the first-order term vanishes if we assume zero-average mistuning, $\sum_{i=1}^{r} z_{i}=0$. Equation (13) can be rewritten more compactly as

$$
s(z)=a \sum_{i=1}^{r} z_{i}+z^{\prime} S z+O\left(\|z\|^{3}\right)
$$

where $\mathscr{S}$ is defined for even and odd $r$, respectively,

$$
S=\left[\begin{array}{cccccccc}
b & \frac{c_{1}}{2} & \frac{c_{2}}{2} & \ldots & c_{k} & \ldots & \frac{c_{2}}{2} & \frac{c_{1}}{2} \\
\frac{c_{1}}{2} & b & \frac{c_{1}}{2} & \frac{c_{2}}{2} & \ldots & c_{k} & \ldots & \frac{c_{2}}{2} \\
\frac{c_{2}}{2} & \frac{c_{1}}{2} & b & \frac{c_{1}}{2} & \frac{c_{2}}{2} & \ldots & c_{k} & \ldots \\
\frac{c_{1}}{2} & \frac{c_{2}}{2} & \ldots & c_{k} & \ldots & \frac{c_{2}}{2} & \frac{c_{1}}{2} & b
\end{array}\right] \text { or }
$$




$$
S=\left[\begin{array}{ccccccccc}
b & \frac{c_{1}}{2} & \frac{c_{2}}{2} & \ldots & \frac{c_{k}}{2} & \frac{c_{k}}{2} & \ldots & \frac{c_{2}}{2} & \frac{c_{1}}{2} \\
\frac{c_{1}}{2} & b & \frac{c_{1}}{2} & \frac{c_{2}}{2} & \ldots & \frac{c_{k}}{2} & \frac{c_{k}}{2} & \ldots & \frac{c_{2}}{2} \\
\frac{c_{2}}{2} & \frac{c_{1}}{2} & b & \frac{c_{1}}{2} & \frac{c_{2}}{2} & \ldots & \frac{c_{k}}{2} & \frac{c_{k}}{2} & \ldots \\
\frac{c_{1}}{2} & \frac{c_{2}}{2} & \ldots & \frac{c_{k}}{2} & \frac{c_{k}}{2} & \ldots & \frac{c_{2}}{2} & \frac{c_{1}}{2} & b
\end{array}\right]
$$

and $z^{\prime}$ denotes the transpose of $z$. Notice that $S$ is real, symmetric, and cyclic, a very special structure caused by the symmetry of the problem.

We now consider our results thus far. By using symmetry arguments, we have reduced find new stability boundaries to find $r / 2+2$ stability coefficients. Once we have found $a, b$, $c_{1}, c_{2}, \ldots, c_{k}$, the analysis problem is solved up to second order in $z$. This is a very useful simplification. Furthermore, we can make very interesting and useful conclusions based on Eq. (14). Some of these conclusions are listed next.

1) The structure proved above is independent of model type; hence, it is true for any model including dynamical system, imposed sinusoidal motion, or CFD models. The only requirement is a $2 \pi / r$ rotation symmetry group.

2) When restricted to zero average, mistuning appears as a second-order effect.

3) To prove Eq. (14) we assumed a rotational symmetry. However, the second-order term, $z^{\prime} S z$ also has a sign and reflection symmetry. In other words, $z^{\prime} S z$ is invariant under $z, \quad-z$ and $\left(z_{1}, z_{2}, \ldots, z_{r}\right), \quad\left(z_{r}, z_{r-1}, \ldots, z_{2}, z_{1}\right)$. This implies that (for a zero-average mistuning) sign and reflection appear as third-order effects. Therefore there is a hierarchy of stability effects; tuned (average) terms appear in the first order, zero average mistuning is a second-order phenomenon, and mistuning reflection is of third order.

The stability coefficients $a, b, c_{1}, \ldots, c_{k}$ remain to be determined, we present two methods to do so in the following subsections.

\section{A. Method A: Computing Eigenvalue Derivatives}

Determining $a, b, c_{1}, \ldots, c_{k}$ can be easily viewed as derivatives of eigenvalues with respect to parameter problems. The approach discussed next has favorable properties, it only requires information at $z=0$ (the tuned case) and is easily adjusted for different types of mistuning with a minimum of computation.

Method A is based on any discrete blade model [Eq. (1)], where the resulting Jacobian

$$
M(U, z) \triangleq \frac{\partial f}{\partial x}\left[x_{0}(U, z), U, z\right]
$$

has the property that the quantities

$$
\begin{array}{ccc}
M\left[U_{\text {crit }}(0), 0\right], & \frac{\partial M}{\partial z_{i}}\left[U_{\text {crit }}(0), 0\right], & \frac{\partial^{2} M}{\partial z_{i} \partial z_{j}}\left[U_{\text {crit }}(0), 0\right] \\
\frac{\partial M}{\partial U}\left[U_{\text {crit }}(0), 0\right], & \frac{\partial^{2} M}{\partial U^{2}}\left[U_{\text {crit }}(0), 0\right], & \frac{\partial^{2} M}{\partial U \partial z_{i}}\left[U_{\text {crit }}(0), 0\right]
\end{array}
$$

can be computed (analytically or numerically) for all $i$ and $j$. Practically, the preceding requirement is not easily satisfied for complex models [Eq. (1)]. Specifically, $M(U, z)$ cannot be computed for most CFD models.

To define $s(z)$ in terms of eigenvalue derivatives, consider the power expansion of $h(U, z)$ (Fig. 3) about $U_{\text {crit }}(0)$ for any $z$ in $\Omega$

$$
\begin{aligned}
& h(U, z)=h\left[U_{\text {crit }}(0), z\right]+\left\{\frac{\partial h}{\partial U}\left[U_{\text {crit }}(0), z\right]\right\}\left[U-U_{\text {crit }}(0)\right] \\
& +\frac{1}{2}\left\{\frac{\partial^{2} h}{\partial U^{2}}\left[U_{\text {crit }}(0), z\right]\right\}\left[U-U_{\text {crit }}(0)\right]^{2}+\mathcal{O}\left[\left|U-U_{\text {crit }}(0)\right|^{3}\right]
\end{aligned}
$$

Figure 3 illustrates a case where eigenvalues cross between $U_{\text {crit }}(0)$ and $U_{\text {crit }}(z)$. However, in assumption 1 , we assume that $s(z)$ is smooth for all $z$ in $\Omega$ which implies that eigenvalues cannot cross, and Eq. (17) holds in $\Omega$. Denote partials with respect to $U$ by subscripts

$$
\begin{gathered}
h(z) \triangleq h\left[U_{\text {crit }}(0), z\right] \\
h_{u}(z) \triangleq \frac{\partial h}{\partial U}\left[U_{\text {crit }}(0), z\right] \\
h_{u u}(z) \triangleq \frac{\partial^{2} h}{\partial U^{2}}\left[U_{\text {crit }}(0), z\right]
\end{gathered}
$$

By definitions of $U_{\text {crit }}$ and $s$ [see Eqs. (4) and (5)], using Eqs. (18-20), and substituting $U=U_{\text {crit }}(z)$ into Eq. (17), we obtain

$$
0=h\left[U_{\text {crit }}(z), z\right]=h(z)+h_{u}(z) s(z)+\frac{1}{2} h_{u u}(z) s^{2}(z)+\mathscr{O}\left(\|z\|^{3}\right)
$$

where the error is derived by noting $s(z)=\mathscr{O}(\|z\|)$.

We can apply identical symmetry arguments to $h(z), h_{u}(z)$, and $h_{u u}(z)$ as we applied to $s(z)$, thus

$$
\begin{gathered}
h(z)=h(0)+\bar{h} \sum_{i=1}^{r} z_{i}+z^{\prime} H z+\mathcal{O}\left(\|z\|^{3}\right) \\
h_{u}(z)=h_{u}(0)+\bar{h}_{u} \sum_{i=1}^{r} z_{i}+z^{\prime} H_{u} z+\mathcal{O}\left(\|z\|^{3}\right) \\
h_{u u}(z)=h_{u u}(0)+\bar{h}_{u u} \sum_{i=1}^{r} z_{i}+z^{\prime} H_{u u} z+\mathcal{O}\left(\|z\|^{3}\right)
\end{gathered}
$$

where $h(0)=0, h_{u}(0), h_{u u}(0), \bar{h}, \bar{h}_{u}, \bar{h}_{u u}$ are constant and the constant matrices $H, H_{u}, H_{u u}$ have the same structure as $S$-real, symmetric, and cyclic.

Substituting Eqs. (14), (22-24) into Eq. (21) yields

$$
\begin{aligned}
& {\left[\bar{h}+a h_{u}(0)\right] \sum_{i=1}^{r} z_{i}+z^{\prime}\left\{H+h_{u}(0) S+\left[\frac{a \bar{h}_{u}+a^{2} h_{u u}(0)}{2}\right] E\right\} z} \\
& \quad+\mathscr{O}\left(\|z\|^{3}\right)=0
\end{aligned}
$$

where $E$ is a full matrix of unit entries that is generated by quadratic cross terms: $\left(\sum_{i=1}^{r} z_{i}\right)^{2}=\boldsymbol{z}^{\prime} E \boldsymbol{z}$. Equation (25) holds for all $z$ in $\Omega$, hence

$$
\bar{h}+a h_{u}(0)=0
$$

and by Lemma B.1

$$
H+h_{u}(0) S+\left[a \bar{h}_{u}+a^{2} h_{u u}(0) / 2\right] E=0
$$

It follows from assumption 1 that $h(U, 0)$ intersects the origin 
at $U_{\text {crit }}(0)$ in a nondegenerate fashion (not as shown in Fig. 4), so $h_{u}(0)=\partial h\left[U_{\text {crit }}(0), 0\right] / \partial u$ is nonzero. Therefore

$$
a=-\frac{\bar{h}}{h_{u}(0)}, \quad S=\frac{1}{h_{u}(0)}\left\{-H+\frac{\bar{h}}{h_{u}(0)}\left[\bar{h}_{u}-\frac{\bar{h} h_{u u}(0)}{2 h_{u}(0)}\right] E\right\}
$$

By definition, $h(U, z)=\operatorname{Re}\left[\boldsymbol{\lambda}_{p}(U, z)\right]$, where $\boldsymbol{\lambda}_{p}$ is the least stable (maximal real part) eigenvalue of $M\left[U_{\text {crit }}(0), 0\right]$. By assumption 1 it remains the least stable eigenvalue for $z$ in $\Theta$ the neighborhood of interest-no eigenvalue switching as in Fig. 5. Using Eqs. (18-20) and differentiating Eqs. (22-24) with respect to $z$ and $U$, yield equations that hold for any $j$ (by symmetry)

$$
\begin{gathered}
\bar{h}=\operatorname{Re}\left\{\frac{\partial \lambda_{p}}{\partial z_{j}}\left[U_{\text {crit }}(0), 0\right]\right\} \\
\zeta=\operatorname{Re}\left\{\frac{\partial^{2} \lambda_{p}}{\partial z_{j}^{2}}\left[U_{\text {crit }}(0), 0\right]\right\} \\
\rho_{i}=\operatorname{Re}\left\{\frac{\partial^{2} \lambda_{p}}{\partial z_{j} \partial z_{j+i}}\left[U_{\text {crit }}(0), 0\right]\right\} \\
h_{u}(0)=\operatorname{Re}\left\{\frac{\partial \lambda_{p}}{\partial U}\left[U_{\text {crit }}(0), 0\right]\right\} \\
\bar{h}_{u}=\operatorname{Re}\left\{\frac{\partial^{2} \lambda_{p}}{\partial U \partial z_{j}}\left[U_{\text {crit }}(0), 0\right]\right\} \\
h_{u u}(0)=\operatorname{Re}\left\{\frac{\partial^{2} \lambda_{p}}{\partial U^{2}}\left[U_{\text {crit }}(0), 0\right]\right\}
\end{gathered}
$$

where $\zeta$ and $\rho_{i}$ are the entries of $H$ and appear in the same format as $b$ and $c_{i}$, the entries of $S$ in Eq. (14). In the preceding equations we can set $j$ to unity for convenience. Substituting Eqs. (29-34) into Eq. (28) yields expressions for the coefficients $a, b, c_{1}, \ldots, c_{k}$ in terms of eigenvalue derivatives

$$
a=-\frac{\left\{\frac{\partial \lambda_{p}}{\partial z_{1}}\right\}}{\left\{\frac{\partial \lambda_{p}}{\partial U}\right\}}
$$

$$
\begin{aligned}
b= & \frac{1}{\left\{\frac{\partial \lambda_{p}}{\partial U}\right\}}-\left\{\frac{\partial^{2} \lambda_{p}}{\partial z_{1}^{2}}\right\} \\
+ & \left.\frac{\left\{\frac{\partial \lambda_{p}}{\partial z_{1}}\right\}}{\left\{\frac{\partial \lambda_{p}}{\partial U}\right\}}\left[\left\{\frac{\partial^{2} \lambda_{p}}{\partial U \partial z_{1}}\right\}-\frac{\left\{\frac{\partial \lambda_{p}}{\partial z_{1}}\right\}\left\{\frac{\partial^{2} \lambda_{p}}{\partial U^{2}}\right\}}{2\left\{\frac{\partial \lambda_{p}}{\partial U}\right\}}\right]\right) \\
c_{i}= & \frac{1}{\left\{\frac{\partial \lambda_{p}}{\partial U}\right\}}\left\{-\left\{\frac{\partial^{2} \lambda_{p}}{\partial z_{1} \partial z_{1+i}}\right\}\right. \\
& +\frac{\left.\partial \frac{\partial \lambda_{p}}{\partial z_{1}}\right\}}{\left\{\frac{\partial \lambda_{p}}{\partial U}\right\}}\left[\left\{\frac{\partial^{2} \lambda_{p}}{\left.\partial U \partial z_{1}\right\}}\left\{\frac{\left\{\frac{\partial \lambda_{p}}{\partial z_{1}}\right\}\left\{\frac{\partial^{2} \lambda_{p}}{\partial U^{2}}\right\}}{2\left\{\frac{\partial \lambda_{p}}{\partial U}\right\}}\right]\right)\right.
\end{aligned}
$$

where all of the preceding derivatives are evaluated at $(U, z)$ $=\left[U_{\text {crit }}(0), 0\right]$, and $\{x\}$ denotes the real part of $x$. It only remains to actually compute the right-hand sides (RHSs) of Eqs. (35-37). These computations are performed using the classic results of Lancaster ${ }^{18}$ for the derivatives of eigenvalues with respect to matrix parameters. Specifically, the symmetric derivatives can be written as

$$
\begin{gathered}
\frac{\partial \lambda_{p}}{\partial \mu}\left[U_{\text {crit }}(0), 0\right]=\rho_{p p} \\
\frac{\partial^{2} \lambda_{p}}{\partial \mu^{2}}\left[U_{\text {crit }}(0), 0\right]=U_{p}^{\prime}\left\{\frac{\partial^{2} M}{\partial \mu^{2}}\left[U_{\text {crit }}(0), 0\right]\right\} \boldsymbol{V}_{p}+2 \sum_{k \in I} \frac{\rho_{p k} \rho_{k p}}{\lambda_{p}-\lambda_{k}}
\end{gathered}
$$

where

$$
\rho_{i j}=\boldsymbol{U}_{i}^{\prime}\left[\frac{\partial M\left[U_{\text {crit }}(0), 0\right]}{\partial \mu}\right] \boldsymbol{V}_{j}
$$

$\boldsymbol{U}_{i}$ and $\boldsymbol{V}_{i}$ are the $i$ th left and right eigenvectors of $M\left[U_{\text {crit }}(0)\right.$, $0]$, the summation is taken over

$$
I=\left\{k: \lambda_{k}\left[U_{\text {crit }}(0), 0\right] \neq \lambda_{p}\left[U_{\text {crit }}(0), 0\right], k \in(1,2, \ldots, r m)\right\}
$$

and $\mu$ can take on the values $z_{1}, z_{2}, \ldots, z_{r}$ or $U$. Using the chain rule we can derive a formula for asymmetric derivatives

$$
\begin{aligned}
& \frac{\partial^{2} \lambda_{p}}{\partial \mu \partial \nu}\left[U_{\text {crit }}(0), 0\right]=\frac{1}{2}\left\{\frac{\partial^{2} \lambda_{p}}{\partial \eta^{2}}\left[U_{\text {crit }}(0), 0\right]-\frac{\partial^{2} \lambda_{p}}{\partial \mu^{2}}\left[U_{\text {crit }}(0), 0\right]\right. \\
& \left.\quad-\frac{\partial^{2} \lambda_{p}}{\partial \nu^{2}}\left[U_{\text {crit }}(0), 0\right]\right\}
\end{aligned}
$$

where $\mu$ and $\nu$ can take on values $z_{1}, z_{2}, \ldots, z_{r}$, or $U$ and $\eta$ are variations in both $\mu$ and $\nu$ (set both $\nu$ and $\mu$ equal to $\eta$ ). This reduces the asymmetric partials to three symmetric partials that can be computed as in Eq. (39). For example, $\partial^{2} \lambda_{p} /$ $\partial z_{1} \partial z_{2}$ is derived by setting $\mu$ to $z_{1}, \nu$ to $z_{2}$, and equating $z_{1}$ and $z_{2}$ to $\eta$, then

$$
\frac{\partial^{2} \lambda_{p}}{\partial \eta^{2}}=\frac{\partial^{2} \lambda_{p}}{\partial z_{1}^{2}}+\frac{\partial^{2} \lambda_{p}}{\partial z_{2}^{2}}+2 \frac{\partial^{2} \lambda_{p}}{\partial z_{1} \partial z_{2}}
$$

Equations $(38-40)$ hold when the eigenvalue $\lambda_{p}\left[U_{\text {crit }}(0), 0\right]$ is simple, meaning that its Jordan block has a simple (diagonal) form. In particular, if $\lambda_{p}\left[U_{\text {crit }}(0), 0\right]$ is distinct then these equations are valid. We do not consider the nondistinct case because Assumption 1 is violated when $\lambda_{p}\left[U_{\text {crit }}(0), 0\right]$ is nondistinct.

At this point it is useful to note when we expect to encounter nondistinct eigenvalues. The symmetry of the problem implies that the Jacobian matrix $M\left[U_{\text {crit }}(0), 0\right]$ has a block circular structure (see Appendix A). Generically, such matrices have distinct eigenvalues and, therefore, generic models will have distinct eigenvalues. However, not all models are generic. For example, the quasisteady model in Appendix A is degenerate because it only includes coupling between adjacent blades and the resulting block circulant Jacobian [Eq. (67)] has only three nonzero blocks. In this case some of the eigenvalues are nondistinct. This leads to two cases:

1) If the least stable eigenvalue is distinct then the fact that other eigenvalues may be nondistinct is irrelevant.

2) If the least stable eigenvalue is not distinct, then these eigenvalues will typically be simple and will travel smoothly with parameters. As a result, Eqs. (38-40) still apply, and so we need to only keep track of multiple eigenvalues to extend the analysis. (In the quasisteady model, only a few of the eigenvalues repeat, and so, the least stable eigenvalue is usually 
distinct. For parameters in this paper, nondistinct least-stable eigenvalues were only observed for $r=6$ or $r=22$.) Notice that the preceding example is actually the exact opposite of what one would expect for unconstrained matrices. If we do not restrict attention to circulant matrices then the class of matrices with nondistinct eigenvalues is generically nonsimple. For a detailed discussion of these issues see Appendix B.

To apply Eqs. (38-40) we need to compute $\boldsymbol{\lambda}_{i}, \boldsymbol{U}_{i}$, and $\boldsymbol{V}_{i}$, the eigenvalues, and the left, right eigenvectors of $M\left[U_{\text {crit }}(0)\right.$, $0]$ for all $i$. Because $M\left[U_{\text {crit }}(0), 0\right]$ has a block circular structure (Appendix A) we can apply the methods of Appendix B to compute the eigenvalues and vectors. These methods provide a significant reduction in computational complexity: instead of solving the $\mathrm{rm} \times \mathrm{rm}$ eigenvalue/vector problem ( $m$ is the number of states per blade), where computation time increases as $(\mathrm{rm})^{3}$, we solve an $m \times m$ eigenvalue/vector problem $r$ times with resulting computation time $\mathrm{rm}^{3}$, a savings of $r^{2}$. These methods also avoid the numerical difficulties inherent in solving eigenvalue/vector problems for matrices with nondistinct eigenvalues.

We conclude this subsection by summarizing the previous procedure for the quasisteady model introduced in Appen$\operatorname{dix}$ A.

Step 1: Derive the matrix $M(U, z)$-Appendix A, Eq. (67).

Step 2: Compute the eigenvalues of $M(U, 0)$ for $U \in\left[U_{0}\right.$, $U_{1}$ ], the range of interest, by the methods of Appendix B.

Step 3: From step 2, construct $h(U, 0)$ the maximal real part of eigenvalues at every $U \in\left[U_{0}, U_{1}\right]$.

Step 4: Find $U_{\text {crit }}(0)$, the point where $h(U, 0)$ changes sign. Suggested methods to do so are a bisection algorithm or the Newton-Raphson method.

Step 5: Compute left and right eigenvectors $\left(\boldsymbol{U}_{i}, \boldsymbol{V}_{i}\right)$ of $M\left[U_{\text {crit }}(0), 0\right]$ using Appendix B.

Step 6: Evaluate Eqs. (38-40) as $\mu$ and $\nu$ vary over $z_{1}, z_{2}$, $\ldots, z_{r}$ and $U$.

Step 7: Substitute the results of step 6 into Eqs. (35-37) to compute the stability coefficients $a, b, c_{1}, \ldots, c_{k}$.

Notice that all of the steps only require information at the tuned point $z=0$. The mathematica code that implements method $\mathrm{A}$ is available by request from the author.

\section{B. Method B: Finite Difference}

For some models [Eq. (1)] it is not possible to compute the quantities of Eq. (16). In particular, it is impractical to compute $M(U, z)$ and its derivatives for CFD models because of their complexity and large number of states.

Given any model [Eq. (1)], which can accurately predict $s(z)$ for any given mistuning $z$, estimate the coefficients $a, b, c_{1}$, $\ldots, c_{k}$ by finite difference. From Eq. (13)

$$
\begin{gathered}
a=\frac{\partial_{s}}{\partial z_{1}}(0) \approx \frac{s(\varepsilon, 0, \ldots, 0)}{\varepsilon} \\
b=\frac{1}{2} \frac{\partial^{2} s}{\partial z_{1}^{2}}(0) \approx \frac{s(2 \varepsilon, 0, \ldots, 0)-2 s(\varepsilon, 0, \ldots, 0)}{2 \varepsilon^{2}} \\
c_{i}=\frac{\partial^{2} s}{\partial z_{1} \partial z_{1+i}}(0) \\
\approx \frac{s(\varepsilon, 0, \ldots, 0, \varepsilon, 0, \ldots, 0)-2 s(\varepsilon, 0, \ldots, 0)}{\varepsilon^{2}}
\end{gathered}
$$

where in Eq. (44) the second $\varepsilon$ in $s(\varepsilon, 0, \ldots, 0, \varepsilon, 0, \ldots, 0)$ appears in the $(i+1)$ th spot and $\varepsilon$ is small. To obtain $a, b$, $c_{1}, \ldots, c_{k}$ we need to run model of Eq. (1) a total of $r / 2+2$ times to form the RHS of Eqs. (42-44). Once these runs have been completed, the analysis question is solved and the effect of all other types of mistuning is known up to second-order in $\boldsymbol{z}$. To estimate the required coefficients we need a model that predicts $s(z)$ accurately so that meaningful second-order finite differences may be formed. Consequently, the preceding method is susceptible to numerical noise, which may cause large errors when attempting to numerically determine secondorder derivatives.

\section{Synthesis}

Once the analysis question has been solved, the next obvious task is synthesis: when is mistuning beneficial? If it is beneficial what is the optimal mistuning? To address these questions define the notion of optimal mistuning.

First it is necessary to decide which type of mistuning will be used. Various possibilities include blade stiffness, blade angle of attack, stator shape, cowling clearance, and many other forms of mistuning. In this section it is assumed that the type of mistuning has been predetermined and we will not concern ourselves with optimizing over type. From a practical standpoint we wish to maximize the stability extension $s(z)$ while keeping the side effects of mistuning acceptable. Here side effects refer to everything from increased weight and manufacturing cost to a decrease in operating range ${ }^{10}$ because of mistuning. To solve the true optimal problem we would quantify all of the possible side effects (such as cost, weight, operating range, and many others) and form a constrained optimization problem where we maximize $s(z)$ subject to the constraint that side effects remain below some practically motivated boundary. Clearly, such an approach is too ambitious. At present we do not know how to quantify increase in cost, operating range, and other factors as a function of mistuning. Furthermore, there is no way to compose a complete list of all possible side effects. Consequently, an optimization problem thus obtained will almost certainly be untractable because of the complexity of constraints motivated by acceptable side effects. To avoid these difficulties, we simply restrict the size of mistuning and assume that resutling side effects are acceptable if $z$ is sufficiently small. In future research we will begin to include physically motivated constraints to account for important side effects.

It remains to define the size of mistuning. To motivate the norm chosen, consider a blade stiffness mistuning. Manufacturing and weight considerations would allow some small variation in each blade, so $\left|z_{i}\right| \leq \varepsilon$ for all $i$. This leads to a natural optimization problem constrained by the infinity norm on $z$ :

3) Optimization: Maximize $s(z)=$ a $\sum_{i=1}^{r} \mathrm{Z}_{i}+z^{\prime} S z+\mathcal{O}\left(\|z\|^{3}\right)$ subject to $\|z\|_{\infty} \leq \varepsilon$.

Having formulated the optimization problem we can determine if mistuning is beneficial. Of course it is understood that we are judging the benefit based on the model chosen to represent the jet engine.

We show that mistuning only makes sense if $\boldsymbol{\varepsilon}$ is sufficiently large compared to a ratio of linear $(a)$ to quadratic $\left(b, c_{1}, \ldots\right.$, $c_{k}$ ) terms in Eq. (13). Recall Eq. (14)

$$
s(z)=a \sum_{i=1}^{r} z_{i}+z^{\prime} S z+\mathscr{O}\left(\|z\|^{3}\right)
$$

where $S$ is a real, symmetric, cyclic matrix containing the quadratic coefficients $b, c_{1}, \ldots, c_{k}$, and $z^{\prime}$ is the transpose of $\boldsymbol{z}$. Let

$$
\bar{z} \quad \text { solution to: } \max a \sum_{i=1}^{r} z_{i} \text { subject to }\|z\|_{\infty} \leq 1
$$

$z^{*} \quad$ solution to: $\max z^{\prime} S z \quad$ subject to $\|z\|_{\infty} \leq 1, \sum_{i=1}^{r} z_{i}=0$

By inspection, $\bar{z}=(1,1, \ldots, 1)$, and corresponds to a mean tuned increase in parameters (assume $a>0$, else reverse sign 


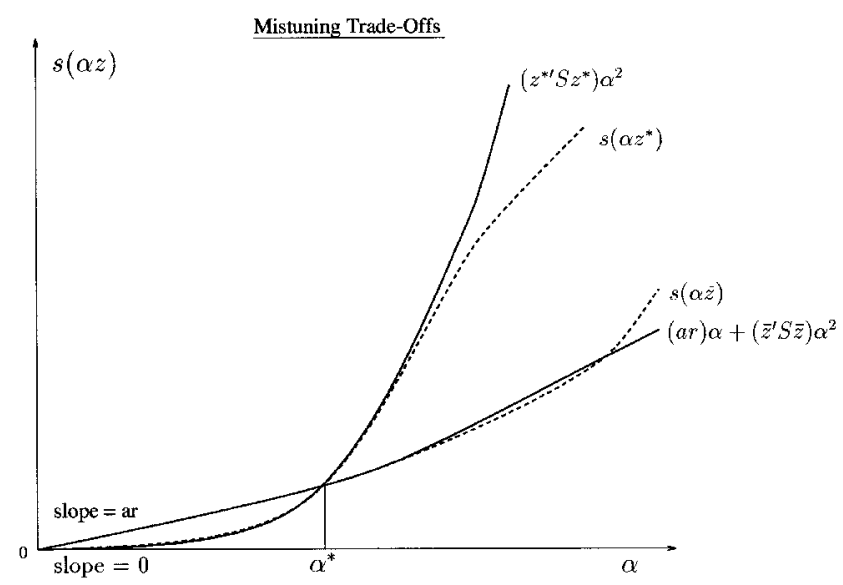

Fig. 6 Worthwhile mistuning lower bound. ----, true stability extension; —-, second-order approximation.

of $\bar{z})$. Conversely, $z^{*}$ corresponds to a zero-average mistuning that optimizes $s(z)$ up to second order (assume $S \neq 0$ and, hence, $z^{*} \neq 0$ ). If we impose a zero-average restriction and truncate third-order terms, then optimization 3 has solution $\varepsilon z^{*}$. Furthermore, $\left\|z^{*}\right\|_{\infty}=1$ else $z^{* \prime} S z^{*}$ may be increased by $z^{*}, \quad(1+\boldsymbol{\delta}) z^{*}$

Now ask the following practically motivated question: given an allowable size of mistuning $\boldsymbol{\varepsilon}$, is it better to apply the optimal zero average mistuning $z^{*}$ or just increase parameters all around by a tuned amount $\bar{z}$ ? So compare

$$
\begin{aligned}
& s(\alpha \bar{z})=(\operatorname{ar}) \boldsymbol{\alpha}+\left(\bar{z}^{\prime} S \bar{z}\right) \alpha^{2}+\mathcal{O}\left(\alpha^{3}\right) \\
& s\left(\alpha z^{*}\right)=0+\left(z^{*} S z^{*}\right) \alpha^{2}+\mathcal{O}\left(\boldsymbol{\alpha}^{3}\right)
\end{aligned}
$$

where $\alpha>0$ is the size of mistuning. For sufficiently small $\alpha$, the tuned stability extension $s(\alpha \bar{z})$ is always greater because it has a nonzero linear term (ar) $\boldsymbol{\alpha}$. However, $\boldsymbol{z}^{*} S z^{*}$ is typically greater than $\bar{z}^{\prime} S \bar{z}$ because $z^{*}$ is the constrained quadratic optimum (note $z^{*} \neq \bar{z}$ ) and, hence $\left(z^{* \prime} S z^{*}\right) \alpha^{2}$ eventually overtakes (ar) $\boldsymbol{\alpha}+\left(\bar{z}^{\prime} S \bar{z}\right) \boldsymbol{\alpha}^{2}$ (Fig. 6). Such a crossover occurs at

$$
\alpha^{*}=\frac{r a}{z^{* \prime} S z^{*}-\bar{z}^{\prime} S \bar{z}}
$$

Based on this second-order analysis: if $\boldsymbol{\alpha}^{*}<\boldsymbol{\varepsilon}$, then $\boldsymbol{\varepsilon}$-sized zero-average mistuning is worthwhile $\left[s\left(\boldsymbol{\varepsilon} z^{*}\right)>s(\boldsymbol{\varepsilon} \bar{z})\right]$, otherwise it is not. Rephrasing, a zero-average mistuning is only worthwhile if it is bigger than $\alpha^{*}$. Of course, the second-order approximation may fail at $\alpha^{*}$ if $\alpha^{*}$ is too large, in which case we cannot make any claims. Notice that $\alpha^{*}$ is small if secondorder coefficients $\left(b, c_{1}, \ldots, c_{k}\right)$ dominate the first-order coefficient $a$.

It is now clear that results in this paper allow the reader to judge when to apply mistuning, based on the model (1). However, they also determine which mistuning should be applied. This is done by solving the optimization 3 up to second order-a standard linear constraint quadratic programming problem. Numerical techniques ${ }^{17}$ exist that can find global maximums of $a \sum_{i=1}^{r} z_{i}+z^{\prime} S z$ subject to $\|z\|_{\infty} \leq \varepsilon$. Current software can usually solve problems up to $r \approx 30$. Larger optimizations take too long ( $r=60$ is projected to take approximately 30 years), but it is possible that computation time can be decreased dramatically by utilizing the special structure of $S$. In fact, because general quadratic programs are provably nonpolynomial time hard, ${ }^{19}$ solutions to large optimal mistuning problems will not be possible unless one exploits the special symmetry and structure of the mistuning problem. Such exploitation of problem structure-and the resulting dramatic decrease in computational complexity-is demonstrated in the next section.

\section{Combinatorial Optimization with Structure}

Mistuning can, at first glance, lead to notoriously difficult optimization problems. For example, suppose $n$ blades are made and these blades have a set of mistuning values (say stiffness variations) $y_{1}, y_{2}, \ldots, y_{n}$ because of machining tolerances. Given the stability coefficients $a, b, c_{1}, c_{2}, \ldots, c_{k}$ and assuming $y_{i}$ are known (measurable), what is the arrangement of the $r$ blades that maximizes stability? If we wanted to solve the problem exhaustively, we would have to check $n$ permute $r$ or $n ! /(n-r)$ ! possibilities. Clearly, this is not practical for large $r$ or $n$; even the special case $n=r$ requires $r$ ! operations. A possible solution lies in the problem structure-exploiting such structure can result in tremendous complexity reduction. Specifically, circular structure of the mistuning problem and the additional structure of the stability coefficients $c_{i}$ found in the quasisteady model (see Sec. VII and Table 1) allows solution of the $n=r$ combinatorial optimization in closed form.

Consider the set of models where stability coefficients satisfy the relation

$$
\begin{gathered}
c_{1}>c_{2}>\cdots>c_{k-1}>c_{k}, \quad r \quad \text { odd } \\
c_{1}>c_{2}>\cdots>c_{k-1}>2 c_{k}, \quad r \quad \text { even }
\end{gathered}
$$

Condition (49) or (50) holds for the quasisteady model (Appendix A) over a fairly broad range of parameter values. There is no reason for this condition to hold for other models, yet there is also no reason to suppose that such structure is specific to the quasisteady model. When condition (49) or (50) holds, the following problem may be solved closed form:

4) Combinatorial Optimization: Given mistuning values $\left(y_{1}, y_{2}, \ldots, y_{r}\right)$, the stability coefficients $\left(a, b, c_{1}, \ldots, c_{k}\right)$ that satisfy condition (49) or (50) and the resulting matrix $S$ of Eq. (14); maximize $s(z)=$ a $\sum_{i=1}^{r} z_{i}+z^{\prime} S z$ subject to $z \in \Pi=\{z$ : $\left.z=\left(y_{l_{1}}, y_{l_{2}}, \ldots, y_{l_{r}}\right), l_{i} \neq l_{j}, \forall i \neq j\right\}$.

Optimization 4 is not restricted to mistuning applications. An identical optimization arises in computer science-related to the optimal arrangement of records to be searched-and its statement and terse solution can be found in $\mathrm{Knuth}^{20}$ (p. 405, Q18 and Q20). Optimal solutions are all rotations and reflections of the pyramid arrangement $z=\left(z_{1}, z_{2}, \ldots, z_{r}\right)$, where $z_{k+1} \geq z_{k} \geq z_{k+2} \geq z_{k-1} \geq \cdots \geq z_{r-1} \geq z_{2} \geq z_{r} \geq z_{1}$ for $r$ even or $z_{k+1} \geq z_{k} \geq z_{k+2} \geq z_{k-1} \geq \cdots \geq z_{2} \geq z_{r-1} \geq z_{1} \geq z_{r}$ for $r$ odd (Fig. 7). Computational complexity drops from $r$ ! to $r \log r$, which is the sorting time for $r$ objects. ${ }^{20}$

The proof presented here follows Knuth. ${ }^{20}$ We show the $r$ odd case but $r$ even is almost identical, the only difference being the factor of 2 that multiplies $c_{k}$ in Eq. (50). First note that the solution to optimization 4 is independent of the coefficients $a$ and $b$ because the relevant terms $a \sum_{i=1}^{r} z_{i}$ and $b \sum_{i=1}^{r} z_{i}^{2}$ are invariant under permutations of $z$. Therefore, without loss of generality, we may consider the objective function $z^{\prime} \mathscr{C} z=z^{\prime}(S-b I) z$, which depends on coefficients $c_{i}$ only. Now, for any $\boldsymbol{z}$ make one of two identifications (here $\boldsymbol{\beta}$ and $\delta$ are placeholder variables)

$$
\begin{aligned}
& z=\left(\boldsymbol{\alpha}_{1}, \boldsymbol{\alpha}_{2}, \ldots, \boldsymbol{\alpha}_{k}, \boldsymbol{\beta}, \gamma_{k}, \ldots, \gamma_{2}, \gamma_{1}\right) \\
& z=\left(\alpha_{1}, \alpha_{2}, \ldots, \boldsymbol{\alpha}_{k}, \gamma_{k}, \ldots, \gamma_{2}, \gamma_{1}, \delta\right)
\end{aligned}
$$

and define the sets

$$
\begin{aligned}
& A=\left\{i: \alpha_{i}<\gamma_{i}, i \in(1,2, \ldots, k)\right\} \\
& B=\left\{i: \alpha_{i}=\gamma_{i}, i \in(1,2, \ldots, k)\right\} \\
& C=\left\{i: \alpha_{i}>\gamma_{i}, i \in(1,2, \ldots, k)\right\}
\end{aligned}
$$


Table 1 Analysis coefficients for stiffness and blade angle-of-attack mistuning

\begin{tabular}{lcccccccccc}
\hline \hline Mistuning & $a$ & $b$ & $c_{1}$ & $c_{2}$ & $c_{3}$ & $c_{4}$ & $c_{5}$ & $c_{6}$ & $c_{7}$ & $c_{8}$ \\
\hline Stiffness & 29.70 & 55.81 & 71.99 & 37.56 & 8.44 & -15.40 & -33.93 & -47.17 & -55.12 & -57.77 \\
Angle & -1.83 & -1.05 & -0.20 & -0.10 & -0.30 & 0.03 & 0.09 & 0.12 & 0.14 & 0.15 \\
\hline \hline
\end{tabular}

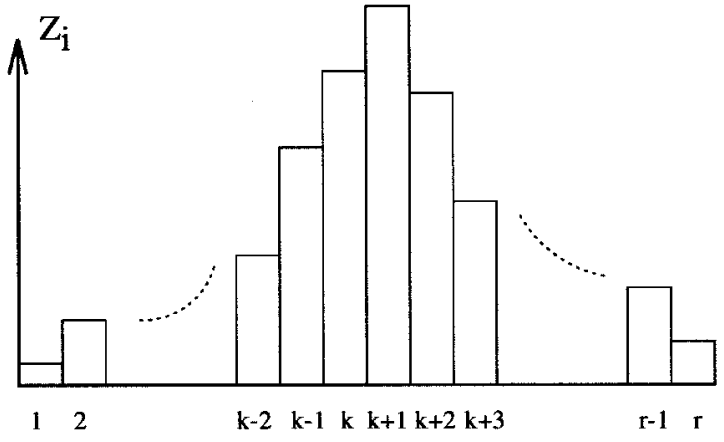

Fig. 7 Optimal pyramid arrangement $(r$ even $)$

Consider switching $\boldsymbol{\alpha}_{i}$ and $\gamma_{i}$ when $\alpha_{i}>\gamma_{i}$; hence, switch for all $i \in C$. For both Eqs. (51) and (52) it can be shown that the resulting change in the objective function $z^{\prime} \mathscr{C}_{z}$ is given by

$$
\Delta=\sum_{i \in C, j \in A}\left[c_{|i-j|}-c_{l(i, j)}\right]\left(\gamma_{j}-\alpha_{j}\right)\left(\alpha_{i}-\gamma_{i}\right)
$$

where

$$
l(i, j)= \begin{cases}i+j-h, & i+j-h \leq k \\ r-(i+j-h), & i+j-h>k 1\end{cases}
$$

and $h$ is 1 or 0 , depending on whether we consider Eqs. (51) or (52), respectively. It follows that $|i-j|<l(i, j)$ for all $i, j$ in $(1,2, \ldots, k)$. Hence, $c_{|i-j|}>c_{l(i, j)}$ by condition (49), and the terms $\left(\gamma_{j}-\alpha_{j}\right)$ and $\left(\alpha_{i}-\gamma_{i}\right)$ are strictly positive by definition of sets $A$ and $C$. Consequently, $\Delta>0$ unless either $A$ or $C$ is empty. In other words, we can improve on $z$ in Eq. (51) or (52) if both $A$ and $C$ are nonempty. It turns out that only the pyramid arrangement of Fig. 7 has either $A$ or $C$ empty for all rotations and reflections; hence, it is the only arrangement that cannot be improved by the construction [Eqs. (49-57)].

Next, recall that optimums come in sets of $2 r$-if $z^{*}$ is an optimum then so is any rotation or reflection of $z^{*}$. [Because a reflection about $z_{i}^{*}$ is equivalent to a reflection about $z_{1}^{*}$ and $2(i-1)$ rotations, we really only have one reflection and $r$ rotations for a total of $2 r$ equivalent optimums.] Suppose an optimal solution $z^{*}$ of optimization 4 is not a rotation or reflection of the pyramid arrangement. Rotate $z^{*}$ so that $z_{p}^{*}=$ $\max _{i} z_{i}^{*}$ appears in the $(k+1)$ th spot (if there is more than one maximum then pick any of these maxima), and reflect $z^{*}$ about $z_{k+1}^{*}$ if $z_{k}^{*}<z_{k+2}^{*}$. These two operations yield an equivalent optimum and imply $z_{k+1}^{*} \geq z_{k}^{*} \geq z_{k+2}^{*}$

Because $z^{*}$ is not a rotation or reflection of the pyramid arrangement then one of the inequalities in the top row of Eq. (58) must fail. The middle and bottom row correspond to the same chain of inequalities using the relabeling of Eqs. (51) and (52), respectively,

$$
\begin{aligned}
& {\left[\begin{array}{c}
z_{k+1}^{*} \\
\boldsymbol{\beta} \\
\gamma_{k}
\end{array}\right] \geq\left[\begin{array}{l}
z_{k}^{*} \\
\boldsymbol{\alpha}_{k} \\
\boldsymbol{\alpha}_{k}
\end{array}\right] \geq\left[\begin{array}{c}
z_{k+2}^{*} \\
\gamma_{k} \\
\gamma_{k-1}
\end{array}\right] \geq\left[\begin{array}{l}
z_{k-1}^{*} \\
\alpha_{k-1} \\
\alpha_{k-1}
\end{array}\right] \geq \cdots \geq\left[\begin{array}{l}
z_{2}^{*} \\
\alpha_{2} \\
\alpha_{2}
\end{array}\right]} \\
& \geq\left[\begin{array}{c}
z_{r-1}^{*} \\
\gamma_{2} \\
\gamma_{1}
\end{array}\right] \geq\left[\begin{array}{c}
z_{1}^{*} \\
\alpha_{1} \\
\alpha_{1}
\end{array}\right] \geq\left[\begin{array}{c}
z_{r}^{*} \\
\gamma_{1} \\
\delta
\end{array}\right]
\end{aligned}
$$

First consider the case where $z_{k+1}^{*} \neq z_{k}^{*} \neq z_{k+2}$; hence, $z_{k+1}^{*}>$ $z_{k}^{*}>z_{k+2}^{*}$. We have assumed $z^{*}$ is not a pyramid arrangement, so at least one of the inequalities in Eq. (58) must fail. There are two possibilities: either $\alpha_{i} \geq \gamma_{i}$ fails in the middle row of Eq. (58) for some $i$ or $\gamma_{j} \geq \alpha_{j}$ fails in the bottom row of Eq. (58) for some $j$. In the first possibility, $\gamma_{i}>\alpha_{i}$, and so $A$ is nonempty, but $z_{k}^{*}>z_{k+2}^{*}$, and so $\alpha_{k}>\gamma_{k}$ and $C$ must be nonempty. Hence, $\Delta$ of Eq. (56) is positive, $z^{*}$ can be improved by the construction of Eqs. (49-57), and so $z^{*}$ is not the optimum-a contradiction! Similarly, possibly two $\alpha_{j}>\gamma_{j}$, so $C$ is nonempty, but $\gamma_{k}>\alpha_{k}$ and, hence, $A$ is nonempty. This also contradicts the assumption that $z^{*}$ is optimal.

Now consider the cases where $z_{k+1}^{*} \neq z_{k}^{*} \neq z_{k+2}$ does not hold When $z_{k+1}^{*}=z_{k}^{*}$ but $z_{k}^{*} \neq z_{k+2}^{*} \neq z_{k-1}^{*}$, then $z_{k}^{*}>z_{k+2}^{*}$ because $z_{k}^{*}$ is a maximum of $z^{*}$ and we may reflect so that $z_{k+2}^{*}>z_{k-1}^{*}$. Now we apply the same arguments as discussed earlier, except $\gamma_{k}>\alpha_{k}$ gets replaced by $\gamma_{k-1}>\alpha_{k-1}$ to ensure $A$ is nonempty for the bottom inequality chain. The same reflection and right shift applies for the next case where $z_{k+1}^{*}=z_{k}^{*}=z_{k+2}^{*} \neq z_{k-1}^{*} \neq$ $z_{k+3}^{*}$, etc. Notice that we need at least two values of $z^{*}$ different from the maximal value $z_{p}^{*}=\max _{i} z_{i}^{*}$, otherwise all arrangements are optimal. For example, if the mistuning values are $y_{1}$ $=y_{2}=\cdots=y_{r-1} \neq y_{r}$, then all arrangements are equivalent by circular symmetry.

\section{Results and Applications to a Simple Quasisteady Model}

Conceptually, there are two levels of results in this paper Level one encompasses a reduction of practical mistuning issues to tractable mathematical questions. This level includes definitions of stability extensions, symmetry arguments, quadratic optimizations, large eigenvalue matrix problems and their reduction to smaller (more tractable) matrix questions. It is stressed that this level is model independent and provides a general framework for analyzing and optimizing mistuning. Of course it is understood that no single method can be general enough to encompass all possible cases. However, the method presented in this paper is quite simple. As a result, for specific applications that may fall outside the scope of level one, it becomes obvious how to extend the analysis in question. For example, if it is found that third-order terms are important in $s(z)$, then the extension required involves computing third-order coefficients and optimizing over cubic terms. Thus, the main contribution of this paper is the consistent, systematic approach to mistuning that is presented.

Level two lies below level one and deals with specific models. Researchers may pick whichever model [Eq. (1)] they believe captures relevant aerodynamic effects for their specific application. Once a specific model has been picked, symmetry arguments presented (or an extension thereof) can be applied to solve the analysis and synthesis questions. It is hoped that methods in this paper will serve as a guiding principle in choice of relevant models. For example, suppose experiments show that mistuning in a given application has a large effect on stability. Then from Sec. V, Eq. (48), $\alpha^{*}=r a /\left(z^{* \prime} S z^{*}-\right.$ $\left.\bar{z}^{\prime} S \bar{z}\right)$ must be small. If the model chosen does not have this property then it follows that this model does not accurately predict mistuning.

For the purposes of this paper, we use a quasisteady model (Appendix A) to present and validate the symmetry arguments. Because this is the simplest model that will display flutter-like instabilities, we urge the reader to treat these results with caution. The model used assumes quasisteady aerodynamics (hence, it does not include unsteady fluid dynamics), there is only one degree of freedom and a single blade coupling 

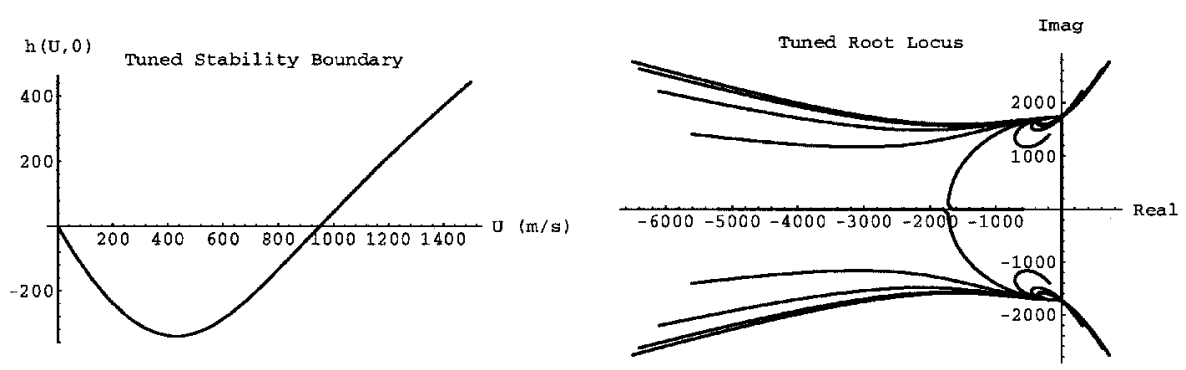

Fig. 8 Quasisteady model: motion of tuned eigenvalues, stability boundary, and root locus.

mechanism. As a result, this model is incapable of capturing complex nonlinear behavior or important unsteady aerodynamic effects. Nevertheless, this model is still quite useful because the blade coupling included captures one of the major causes of instabilities in blade cascades.

Within the model, we can mistune three quantities, they are stiffness $k_{i}$, mass $m_{i}$, and blade angle of attack $\boldsymbol{\beta}_{i}$. It was noticed that mass and stiffness mistuning result in almost identical behavior, both in coefficients computed $a, b, c_{1}, \ldots, c_{k}$ and in optimization results. Consequently, we only discuss stiffness and angle-of-attack mistuning. At the end of Sec. IV.A, we outlined seven steps to compute the coefficients $a$, $b, c_{1}, \ldots, c_{k}$. To illustrate results (which were found to be typical across parameter space), we pick parameters in the quasisteady model corresponding to a high-speed fan with 16 blades $(r=16)$. Computing eigenvalues and vectors by the methods of Appendix B we can plot $h(U, 0)$ of step 3, Sec. IV.A and a tuned root locus plot (Fig. 8). Hence, $U_{\text {crit }}(0)=$ $950.4 \mathrm{~m} / \mathrm{s}$. (Here we see the first drawback of the quasisteady model, it cannot capture compressible effects and the instability velocity is far too high.) Evaluating steps 5-7 (end of Sec. IV.A) yields the coefficients $a, b, c_{1}, \ldots, c_{k}$. Comparison with Method B of Sec. IV.B shows that for $\varepsilon$ sufficiently small in Eqs. (42-44), the coefficients computed by methods A and B fall arbitrarily close to one another (up to machine error); hence, results for method B are not shown.

For angle-of-attack mistuning, it can be shown in closed form (Appendix B) that $S$ [of Eq. (14)] is negative definite. As a result, for zero-average mistuning we have $s(z) \approx z^{\prime} S z<0$ for all $z \neq 0$ [see Eq. (14)]. Hence, the quasisteady model predicts that zero-average, angle-of-attack mistuning can only decrease stability.

In the case of stiffness mistuning, it was found that $\boldsymbol{\alpha}^{*}=$ 0.1 in Eq. (48). Note that $\alpha^{*}$ is the crossover where optimal zero-average mistuning (accurate to second order) first surpasses a tuned increase; hence, it makes no sense to mistune by less than $\alpha^{*}$. However, the second-order approximation of Eq. (14) fails at $\alpha \approx 0.08$. Within the second-order range, we must conclude that mistuning is not beneficial. Outside the second-order range we cannot make any claims based on our second-order analysis.

We compute stability outside the second-order range by brute force. Recall the definitions of the tuned increase and optimal zero-average mistuning, $\bar{z}$ and $z^{*}$, defined after Eq. (45). If we compute $s(\alpha \bar{z})$ and $s\left(\alpha z^{*}\right)$ as shown in Eqs. (46) and (47), we then see that $s(\alpha \bar{z})$ continues to increase beyond $\alpha=0.08$ but $s\left(\alpha z^{*}\right)$ falls sharply beyond 0.08 (this computation was based on solving for the eigenvalues directly instead of approximating them to second order). Thus, $s(\alpha \bar{z})$ is always greater than $s\left(\alpha z^{*}\right)$, and so the quasisteady model predicts that second-order optimal zero-average mistuning cannot beat a tuned mean increase. Yet, based on experimental results such as Ref. 6, we expect mistuning to have a large effect on stability, certainly much larger then would be caused by a small tuned increase in stiffness. Our conclusion (modulo obvious considerations such as, mistuning does not have a large effect at these parameters) must be that the quasisteady model is a

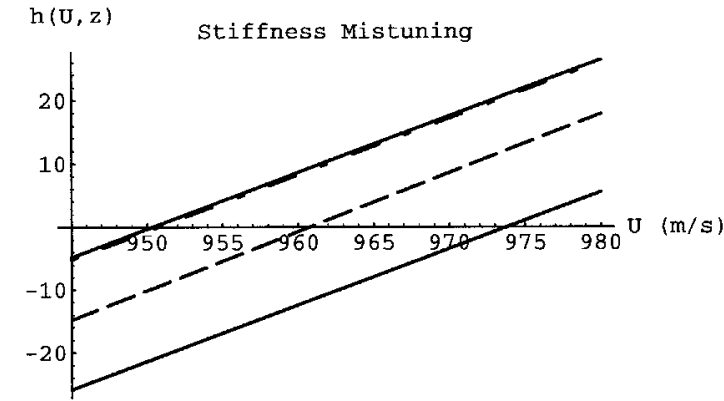

Fig. 9 Hierarchy of mistuning boundaries based on quasisteady model.

poor predictor of mistuning, which is not surprising considering its simplicity.

To determine the optimal zero-average stiffness mistuning, we numerically solve the optimization problem 3 up to second order, subject to the additional constraint $\sum_{i=1}^{r} z_{i}=0$. The optimal solution $\varepsilon z^{*}$ has the form $z^{*}=(1,1, \ldots, 1,-1,-1$, $\ldots,-1)$. In other words, if we have $r$ stiffnesses for $r$ blades, denoted $k_{1}$ through $k_{r}$, the optimal zero-average mistuning is in the first mode: $k_{1}=k_{2}=\cdots=k_{r / 2}=k_{0}(1+\varepsilon)$ and $k_{r / 2+1}=$ $\cdots=k_{r}=k_{0}(1-\varepsilon)$. (Recall that $z^{\prime} S z$ has a rotational, reflection, and sign symmetry-see Sec. IV-hence, any rotation, reflection, or sign change of $\varepsilon z^{*}$ is also optimal). Note that the form of the optimal mistuning is very different from the alternate blade mistuning proposed in Ref. 16 , where $k_{1}=k_{3}=$ $\cdots=k_{r-1}=k_{0}(1+\varepsilon)$ and $k_{2}=k_{4}=\cdots=k_{r}=k_{0}(1-\varepsilon)$. It is predicted that the optimal approach is an order of magnitude better than alternate blade mistuning. Figure 9 shows the appropriate stability boundaries for a $5 \%$ mistuning of stiffness. The solid line on the far left represents the tuned stability boundary, it intersects the $U$ axis at $U_{\text {crit }}(0)=950.4 \mathrm{~m} / \mathrm{s}$. Immediately to the right (dash-dot) is the alternate mistuning boundary, further right we find the optimal zero-average stability boundary (dash-dash) and finally, on the far right (solid), we have the tuned increase. There are two things to notice in Fig. 9. First, it makes no sense to introduce mistuning based on the quasisteady model, because the tuned increase is clearly superior to the optimal zero average. Second, there is a huge difference betwen alternate zero-average mistuning and optimal zero-average mistuning. Intuitively, alternate mistuning breaks symmetry only mildly. It reduces the single blade spacing $2 \pi / r$ symmetry to a two-blade spacing $4 \pi / r$ symmetry between even and odd blades. By comparison, first mode mistuning destroys all circumferential symmetry.

Finally, the optimal mistuning problem seems to have a robust structure. Small changes in system parameters and/or operating conditions such as nominal rotor stiffness, blade mass, and nominal angle of attack do not change the optimal answer. Even though the coefficients $a, b, c_{1}, \ldots, c_{k}$ change slightly, their structure remains the same and the optimal solution remains unchanged. Such robustness issues will be addressed rigorously in future research. 


\section{Conclusions}

Results are presented for analyzing and optimizing the stability extensions caused by mistuning. Using mild smoothness conditions and simple symmetry arguments, tough practical issues are reduced to standard, tractable, mathematical problems. Analysis and synthesis problems are solved up to second order in mistuning. Methods are presented to judge when mistuning should be applied. Furthermore, the simplifications presented here allow a researcher to judge easily if his or her model can accurately predict mistuning effects. These techniques are applied to a simple quasisteady model to illustrate and validate the arguments used. One can conclude that this very simple quasisteady model does not predict mistuning effects accurately.

\section{Appendix A: Simple Quasisteady Model}

In this section we introduce the simplest blade cascade model that will go unstable. The primary purpose of this model is to demonstrate the methods in this paper. However, one can draw some interesting, if tentative, conclusions based on this model.

Given a cascade of $r$ blades, assume that each blade (numbered by index $i$ ) has one degree of freedom, namely blade bending $q_{i}$ (see Fig. A1). Structural coupling is modeled by a linear spring with stiffness $k_{i}$. Incoming velocity $U$ is constant between blades, but $\boldsymbol{\beta}_{i}$ is allowed to vary. As a result, we can mistune three quantities: stiffness $k_{i}$, mass $m_{i}$, and blade angle of attack that translates to varying $\boldsymbol{\beta}_{i}$.

Let $x_{i}$ denote displacement of the $i$ th blade from neutral spring position. Then

$$
m_{i} \ddot{x}_{i}+\xi \dot{x}_{i}+k_{i} x_{i}=\mathscr{A}_{i}
$$

where $k_{i} x_{i}$ captures the structural force, and $\mathscr{A}$ is a combination of quasisteady aeroforces.

Aerodynamic forces are modeled in two parts. Part one deals with quasisteady aeroforces on the $i$ th blade because of its own motion, this force is labeled $\mathscr{F}_{i}$ and is derived in standard fashion

$$
\mathscr{F}_{i}=q_{\infty} c C_{L_{\alpha}} \gamma_{i}
$$

where $q_{\infty} \triangleq \rho U^{2} / 2$ is the dynamic pressure, $C_{L_{\alpha}}$ is the lift curve slope, $c$ is the chord, and $\gamma_{i}$ is the effective angle of attack because of blade motion. To complete the description of $\mathscr{F}_{i}$ it remains to compute the angle $\gamma_{i}$. This angle is the flow angle that blade $i$ experiences as a result of its own motion, or the

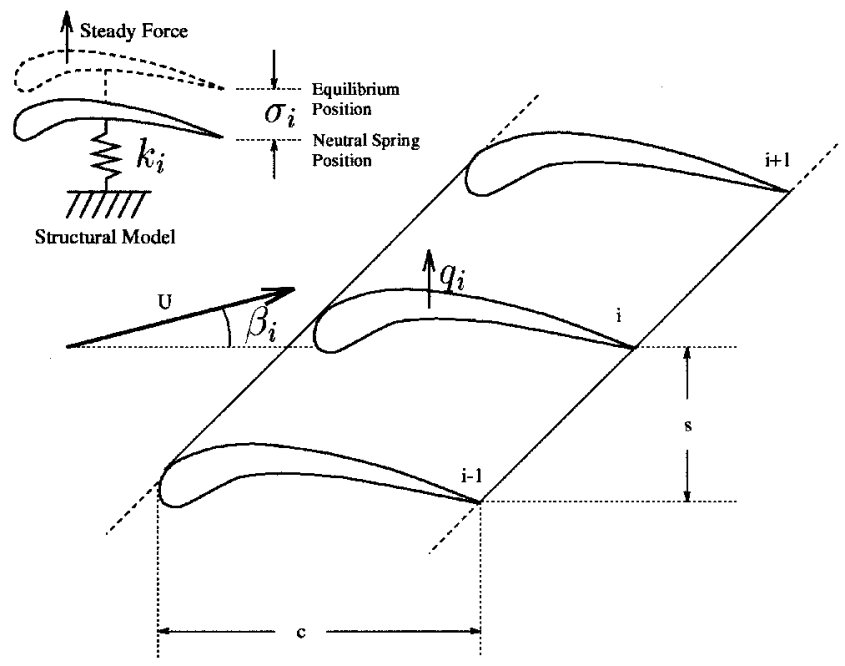

Fig. A1 Cascasde of blades. flow angle in the blade frame of reference. For small $\dot{x}_{i}$, Eq. (60) becomes

$$
\mathscr{F}_{i}=q_{\infty} c C_{L_{\alpha}}\left(\beta_{i}-\frac{\dot{x}_{i} \cos \beta_{i}}{U}\right)
$$

Part two of the aerodynamic forces deals with forces caused by blade coupling. It is assumed that the extra loading on blade $i$ is proportional to the amount of extra flow it must turn, which is in turn proportional to the distance between the $(i-1)$ th and $(i+1)$ th blade. We label this force as $\mathscr{G}$

$$
\mathscr{G}_{i}=p q_{\infty} c\left[\frac{x_{i+1}-x_{i-1}}{2 s}\right]
$$

where $p$ is the coefficient of loading to extra flow turned, and $q_{\infty}, c$, and $s$ play the role of appropriate normalization coefficients. Combining Eqs. (A1), (A3), and (A4) and noting $\mathscr{A}=$ $\mathscr{F}+\mathscr{G}$, yields

$$
\begin{aligned}
& m_{i} \ddot{x}_{i}+\xi \dot{x}_{i}+k_{i} x_{i}=q_{\infty} c C_{L_{\alpha}}\left(\beta_{i}-\frac{\dot{x}_{i} \cos \beta_{i}}{U}\right) \\
& +p q_{\infty} c\left[\frac{x_{i+1}-x_{i-1}}{2 s}\right]
\end{aligned}
$$

Let $\sigma_{i}$ denote the equilibrium position, as shown in Fig. 1A. Setting time derivatives to zero in Eq. (A5) gives

$$
k_{i} \sigma_{i}=q_{\infty} c C_{L_{\alpha}} \beta_{i}+p q_{\infty} c\left[\frac{\sigma_{i+1}-\sigma_{i-1}}{2 s}\right]
$$

If the cascade is tuned $\left(k_{i}=k_{0}, \beta_{i}=\beta_{0}, \forall i\right)$ then Eq. (A6) collapses to $\sigma_{0}=q_{\infty} c C_{L_{\alpha}} \beta_{0} / k_{0}$ because all of the $\sigma$ must equal. However, if the system is not tuned, then the equilibrium changes and $\sigma_{i} \neq q_{\infty} c C_{L_{\alpha}} \beta_{0} / k_{0}$. Therefore, it is once again noted that the relevant equilibrium point does vary as a function of mistuning. (If the reader is concerned about the existence of an equilibrium solution, note that the vector of $\boldsymbol{\sigma}$ satisfies $A \boldsymbol{\sigma}$ $=\boldsymbol{b}$ and it can be shown that $A$ is invertible.)

Now let $q_{i} \triangleq x_{i}-\sigma_{i}$, then Eq. (A5) becomes

$m_{i} \ddot{q}_{i}+\xi \dot{q}_{i}+k_{i} q_{i}=-q_{\infty} c C_{L_{\alpha}} \frac{\cos \beta_{i}}{U} \dot{q}_{i}+p q_{\infty} c\left[\frac{q_{i+1}-q_{i-1}}{2 s}\right]$

Equation (A7) can be rewritten in desired form as

$$
\dot{\boldsymbol{x}}=M(U, z) \boldsymbol{x}
$$

where $\boldsymbol{x}$ is redefined as $\boldsymbol{x}=\left[q_{1}, \dot{q}_{1}, q_{2}, \dot{q}_{2}, \ldots, q_{r}, \dot{q}_{r}\right]$

$$
\begin{gathered}
M(U, z)=\left[\begin{array}{cccccc}
A_{1} & B_{1} & 0 & \ldots & 0 & -B_{1} \\
-B_{2} & A_{2} & B_{2} & 0 & \cdots & 0 \\
& \ddots & \ddots & \ddots & \ddots & \\
B_{r} & 0 & \cdots & 0 & -B_{r} & A_{r}
\end{array}\right] \\
A_{i}\left(U, z_{i}\right)=\left[\begin{array}{ccc}
0 & & \\
-\frac{k_{i}}{m_{i}} & -\frac{\xi}{m_{i}}-\frac{c}{m_{i}} C_{L_{\alpha}} \cos \beta_{i} \frac{q_{\infty}}{U}
\end{array}\right] \\
B_{i}\left(U, z_{i}\right)=\left[\begin{array}{cc}
0 & 0 \\
\frac{p q_{\infty} c}{2 m_{i} s} & 0
\end{array}\right]
\end{gathered}
$$


or $\boldsymbol{\beta}_{i}=\boldsymbol{\beta}_{0}\left(1+\boldsymbol{z}_{i}\right)$. Note that $M(U, z)$ is independent of the equilibrium vector $\left[\sigma_{1}, \ldots, \sigma_{r}\right]$ because the quasisteady model is linear.

If we consider more general models, $M(U, 0)$ will still be of block circular structure as in Eq. (A9). Here, the $A$ matrices along the diagonal reflect forces on blades caused by their own motion, while $B$ is a coupling term between adjacent blades. In this case coupling of the upper blade enters as $B$ while the lower blade generates a symmetric force $-B$. More generally, we can have $B_{\text {up }} \neq-B_{\text {down }}$, and coupling between blades two, three, or four apart would appear as $C_{\text {up }}, C_{\text {down }}, D_{\text {up, }}, D_{\text {down }}, E_{\text {up }}$, $E_{\text {up }}, E_{\text {down }}$, etc. The size of $M(U, z)$ will be states per blade $m$, times the number of blades $r$, for a total of $m \times r$. The circular structure of $M(U, 0)$ in Eq. (A9) is a result of the circumferential symmetry of the problem and will be present for any discrete blade model.

\section{Appendix B: Eigenvalues and Eigenvectors of Block Circular Matrices}

Motivated by Appendix A we consider block circular matrices and derive some standard results. Let $P \in \mathbb{R}^{m \times r m}$ be defined as

$$
P=\left[\begin{array}{cccccc}
P_{1} & P_{2} & P_{3} & \cdots & P_{r-1} & P_{r} \\
P_{r} & P_{1} & P_{2} & P_{3} & \cdots & P_{r-1} \\
& \ddots & & \ddots & & \\
P_{2} & P_{3} & \cdots & P_{r-1} & P_{r} & P_{1}
\end{array}\right]
$$

where $P_{j} \in \mathbb{R}^{m \times m}$ for all $j$. Denote powers of the $r$ th root of unity as $p_{j}=\exp (2 \pi i j / r)$, here $i=\sqrt{-1}$. Now let

$$
\begin{array}{r}
Q_{j}=P_{1}+p_{j} P_{2}+p_{j}^{2} P_{3}+\cdots+p_{j}^{r-1} P_{r} \in \mathbb{C}^{m \times m} \\
\quad j \in(1,2, \ldots, r)
\end{array}
$$

The following theorem allows us to express the eigenvalues and vectors of $P$ as eigenvalues and vectors of $Q_{i}$. Consequently, instead of solving the $\mathrm{rm} \times \mathrm{rm}$ eigenvalue/vector problem where computation time increases as $(\mathrm{rm})^{3}$, we can solve an $m \times m$ eigenvalue/vector problem $r$ times with resulting computation time $\mathrm{rm}^{3}$, a savings of $r^{2}$.

Theorem B.1 For $P$ a block circular matrix as above, let $\lambda_{j}^{d}$, $\boldsymbol{u}_{j}^{d}$, and $\boldsymbol{v}_{j}^{d}$ be the $d$ th eigenvalue, left eigenvector and right eigenvector, respectively, of $Q_{j}$. Then $\lambda_{j}^{d}$ form the eigenvalues of $P$ with left and right eigenvectors $\boldsymbol{U}_{j}^{d}=\left[\boldsymbol{u}_{j}^{d}, p_{j}^{r-1} \boldsymbol{u}_{j}^{d}, p_{j}^{r-2} \boldsymbol{u}_{j}^{d}\right.$, $\left.\ldots, p_{j} \boldsymbol{u}_{j}^{d}\right]$ and $\boldsymbol{V}_{j}^{d}=\left[\boldsymbol{v}_{j}^{d^{\prime}}, p_{j} \boldsymbol{v}_{j}^{d^{\prime}}, \ldots, p_{j}^{r-2} \boldsymbol{v}_{j}^{d^{\prime}}, p_{j}^{r-1} \boldsymbol{v}_{j}^{d^{\prime}}\right]^{\prime}$.

Proof: To prove the theorem we need only show $P \boldsymbol{V}_{j}^{d}=$ $\lambda_{j}^{d} \boldsymbol{V}_{j}^{d}$ and $\boldsymbol{U}_{j}^{d} P=\lambda_{j}^{d} \boldsymbol{U}_{j}^{d}$. Both statements are verified trivially be substitution.

It follows from the theorem that block circular matrices have distinct eigenvalues generically. This is trivial to see for the scalar block case $(m=1)$ because the eigenvalues are simply the $Q_{j}$. Hence, for nondistinct eigenvalues we must have $Q_{i}=$ $Q_{j}$ for $i \neq j$ - an extra condition on the $P_{i}$ that will not hold in general. For block circular matrices the same condition applies, $Q_{i}=Q_{j}$ for $i \neq j$ implies nondistinct eigenvalues. However, in the latter case we can have nondistinct eigenvalues when a particular $Q_{j}$ has nondistinct eigenvalues or if an eigenvalue of $Q_{j}$ equals an eigenvalue of $Q_{i}$ for $Q_{i} \neq Q_{j}$. Both additional cases are also degenerate and, hence, circular matrices have distinct eigenvalues generically. In other words, if we generate block circulant matrices at random then the set of matrices with nondistinct eigenvalues forms a measure zero set.

However, models are not generated at random, and some common model structures lead to degenerate matrices. For ex- ample, if we have a model with no blade coupling then the resulting degenerate circular Jacobian would have the form $P_{1}$ $\neq 0$ and $P_{2}=P_{3}=\cdots=P_{r}=0$, in which case $Q_{1}=Q_{2}=\cdots$ $=Q_{r}=P_{1}$ and all of the eigenvalues are nondistinct. Another example is the quasisteady model used in Appendix A. This model is degenerate because it only includes coupling between adjacent blades, and so, $P_{1} \neq 0,-P_{2}=P_{r} \neq 0$ while $P_{3}=P_{4}$ $=\cdots=P_{r-1}=0$. Hence, some of the $Q_{j}$ repeat and there are some nondistinct eigenvalues. It is important to realize that in these cases the nondistinct eigenvalues are generically simple (have diagonal Jordan form) and so travel smoothly with parameters. This is because each of the $Q_{j}$ will have distinct eigenvalues generically. As a result, each $Q_{j}$ will typically have a complete set of left and right eigenvectors $\left\{\boldsymbol{u}_{j}^{d}, \boldsymbol{v}_{j}^{d}\right\}_{d=1}^{m}$, and so, $P$ will have a complete set of left and right eigenvector $\left\{\boldsymbol{U}_{j}^{d}, \boldsymbol{V}_{j}^{d}\right\}_{j, d=1}^{r, m}$. Notice that unlike the block matrices $Q_{j}$, the eigenvectors $\boldsymbol{U}_{j}^{d}, \boldsymbol{V}_{j}^{d}$ will not repeat. So in the case where $Q_{j}$ repeat we will have nondistinct but typically simple eigenvalues that travel smoothly with parameters. If it happens that the least stable eigenvalue is one of the nondistinct eigenvalues then the only analysis extension required is to keep track of multiple eigenvalues.

In the final degenerate case, where one of the $Q_{j}$ is nonsimple, the eigenvalues may travel discontinuously with parameters, here the analysis fails and cannot be easily extended. We do not expect to encounter this case in practice because it is a measure zero set and there is no expected model structure that would enforce a nonsimple $Q_{j}$.

To conclude this section we state a technical lemma required in Sec. IV whose proof follows easily from properties of symmetric matrices and is not shown.

Lemma B.1 If $R \in \mathbb{R}^{n \times n}$ is a real symmetric matrix, $\Gamma$ is any open neighborhood in $\mathbb{R}^{n}$ about the origin, then $z^{\prime} R z=0, \forall z$ $\in \Gamma$ if and only if $R=0$.

\section{Acknowledgments}

This work was supported by a NSF fellowship and the U.S. Air Force Office of Scientific Research Grant F-49620-95-10409. This work was performed at United Technologies Research Center during the summer of 1996. The author would like to thank M. Myers, D. Gysling, S. Copeland, G. Hendricks, and M. Barnett for their willingness to answer questions at inopportune moments. Suggestions and advice from my advisor, R. Murray, are gratefully acknowledged. Thanks also go to F. Al-Khayyal and T. Van Voorhis at Georgia Institute of Technology for their time and numerical optimization code. Numerical solutions would not have been possible without their help. Software was supplied by F. Al-Khayyal, T. Van Voorhis, and CPLEX Optimization, Inc.

\section{References}

${ }^{1}$ Bendiksen, O. O., "Recent Developments in Flutter Suppression Techniques for Turbomachinery Rotors," Journal of Propulsion and Power, Vol. 4, No. 2, 1986, pp. 164-171.

'Greitzer, E. M., "The Stability of Pumping Systems - The 1980 Freeman Scholar Lecture," Journal of Fluids Engineering, Vol. 103 , June 1981, pp. 193-242.

Bendiksen, O. O., "Flutter of Mistuned Turbomachinery Rotors," Journal of Engineering for Gas Turbines and Power, Vol. 106, Jan. 1984, pp. 25-33.

${ }^{4}$ Crawley, E. F., and Hall, K. C., "Optimization and Mechanisms of Mistuning in Cascades," Transactions of American Society of Mechanical Engineers, Vol. 107, April 1985, pp. 418-426.

5Kaza, K. R., and Kielb, R. E., "Flutter and Response of a Mistuned Cascade in Incompressible Flow," AIAA Journal, Vol. 20, Aug. 1982, pp. $1120-1127$.

Whitehead, D. S., "Torsional Flutter of Unstalled Cascade Blades at Zero Deflection," Aeronautical Research Council, R\&M 3429, March 1964. 
DDye, R. C. F., and Henry, T. A., "Vibration Amplitudes of Compressor Blades Resulting from Scatter in Blade Natural Frequencies," Journal of Engineering for Power, Vol. 91, July 1969, pp. 182-188.

"El-Bayoumy, L. E., and Srinivasan, A. V., "Influence of Mistuning on Rotor-Blade Vibrations," AIAA Journal, Vol. 13, No. 4, 1975, pp. 460-464.

"Whitehead, D. S., "Effect of Mistuning on the Vibration of Turbomachine Blades Induced by Wakes," Journal of Mechanical Engineering Science, Vol. 8, March 1966, pp. 15-21.

${ }^{10}$ Srinivasan, A. V., and Frye, H. M., "Effects of Mistuning on Resonant Stresses of Turbine Blades," Structural Dynamic Aspects of Bladed Disk Assemblies, American Society of Mechanical Engineers, New York, 1976.

${ }^{11}$ Dugundji, J., and Bundas, D. J., "Flutter and Forced Response of Mistuned Rotors Using Standing Wave Analysis," AIAA Journal, Vol. 22, No. 11, 1984, pp. 1152-1161.

${ }^{12}$ Whitehead, D. S., "Force and Moment Coefficients for Vibrating Airfoils in Cascade," Aeronautical Research Council, R\&M 3254, 1960 .

${ }^{13}$ Bloemhof, H., "Flutter of Mistuned Cascades with Structural Coupling," Unsteady Aerodynamics and Aeroelasticity of Turboma- chines and Propellers, 1987.

${ }^{14}$ Pierre, C., and Dowell, E. H., "Localization of Vibrations by Structural Irregularity," Journal of Sound and Vibrations, Vol. 114, No. 3, 1987, pp. 549-564.

${ }^{15}$ Pierre, C., and Murthy, D. V., "Aeroelastic Modal Characteristics of Mistuned Blade Assemblies: Mode Localization and Loss of Eigenstructure," AIAA Journal, Vol. 30, No. 10, 1992, pp. 2483-2496.

${ }^{16}$ Nissim, E., and Haftka, R. T., "Optimization of Cascade Blade Mistuning, Part II: Global Optimum and Numerical Optimization," AIAA Journal, Vol. 23, No. 9, 1985, pp. 1402-1410.

${ }^{17}$ Al-Khayyal, F. A., Larsen, C., and Van Voorhis, T., "A Relaxation Method for Nonconvex Quadratically Constrained Quadratic Programs," Journal of Global Optimization, Vol. 6, No. 3, 1995, pp. 215230.

${ }^{18}$ Lancaster, P., “On Eigenvalues of Matrices Dependent on a Parameter," Numerische Mathematik, Vol. 6, May 1964, pp. 377-387.

${ }^{19}$ Murty, K. G., and Kabadi, S. N., "Some NP-Complete Problems in Quadratic and Nonlinear Programming," Mathematical Programming, Vol. 39, 1987, pp. 117-129.

${ }^{2}$ Knuth, D. E., "Sorting and Searching," The Art of Computer Programming, Vol. 3, Addison-Wesley, Reading, MA, 1973. 